\title{
A degenerate fourth-order parabolic equation modeling Bose-Einstein condensation. Part II: Finite-time blow-up
}

\author{
Ansgar Jüngel* \\ Institute for Analysis and Scientific Computing, Vienna University of Technology, \\ Wiedner Hauptstraße 8-10, 1040 Wien, Austria \\ Michael Winkler\# \\ Institut für Mathematik, Universität Paderborn, \\ 33098 Paderborn, Germany
}

\begin{abstract}
A degenerate fourth-order parabolic equation modeling condensation phenomena related to BoseEinstein particles is analyzed. The model is a Fokker-Planck-type approximation of the BoltzmannNordheim equation, only keeping the leading order term. It maintains some of the main features of the kinetic model, namely mass and energy conservation and condensation at zero energy. The existence of local-in-time weak solutions satisfying a certain entropy inequality is proven. The main result asserts that if a weighted $L^{1}$ norm of the initial data is sufficiently large and the initial data satisfies some integrability conditions, the solution blows up with respect to the $L^{\infty}$ norm in finite time. Furthermore, the set of all such blow-up enforcing initial functions is shown to be dense in the set of all admissible initial data. The proofs are based on approximation arguments and interpolation inequalities in weighted Sobolev spaces. By exploiting the entropy inequality, a nonlinear integral inequality is proved which implies the finite-time blow-up property.
\end{abstract}

Key words: Degenerate parabolic equation, fourth-order parabolic equation, blow-up, weak solutions, entropy inequality, Bose-Einstein condensation, weighted spaces.

MSC 2010: 35K35, 35K65, 35B44, 35B09, 35Q40.

*juengel@tuwien.ac.at

\#michael.winkler@math.uni-paderborn.de 


\section{Introduction}

In this paper, we continue our work [13] in which we have shown the local-in-time existence of weak solutions to the problem

$$
\left\{\begin{aligned}
u_{t}=x^{-\beta}\left(x^{\alpha} u^{n+2}\left(u^{-1}\right)_{x x}\right)_{x x}, & x \in \Omega, t>0, \\
x^{\alpha} u^{n+2}\left(u^{-1}\right)_{x x}=\left(x^{\alpha} u^{n+2}\left(u^{-1}\right)_{x x}\right)_{x x}=0, & x=0, t>0, \\
u_{x}=u_{x x x}=0, & x=L, t>0, \\
u(x, 0)=u_{0}(x), & x \in \Omega,
\end{aligned}\right.
$$

where $\alpha \geq 0, \beta \in \mathbb{R}, n>0$, and $\Omega=(0, L) \subset \mathbb{R}$. This model describes the evolution of the energy distribution $u(x, t)$ as a function of the energy $x \geq 0$ and time $t>0$ in a particle system. The boundary conditions at zero energy $x=0$ are of no-flux type, whereas the boundary conditions at $x=L$ model the fact that the number of particles with (very) large energies is negligible.

The PDE in (1.1) is a Fokker-Planck approximation of the Boltzmann-Nordheim equation, modeling the dynamics of weakly interacting quantum particles like bosons [11. The physical parameters are given by $\alpha=\frac{13}{2}, \beta=\frac{1}{2}$, and $n=2$ but we allow for more general values. The approximation maintains some of the features of the original Boltzmann equation. In particular, it conserves the total mass $N=\int_{\Omega} x^{\beta} u d x$ and the kinetic energy $E=\int_{\Omega} x^{\beta+1} u d x$. The Boltzmann-Nordheim equation admits solutions which blow up in finite time if the initial density is sufficiently dense, modeling the condensation process [6, 7]. The question arises if the local approximation underlying (1.1) also possesses this feature. In this paper, we prove that this is indeed the case under appropriate conditions.

Because of the high complexity of the Boltzmann-Nordheim equation, approximate Fokker-Planck-type equations modeling condensation phenomena related to Bose-Einstein particles were investigated in the literature. The above equation is one of these approximations. Other approximations include the Kompaneets equation [16], the Fokker-Planck model of Kaniadakis and Quarati [14, and the superlinear drift equation of Carrillo et al. [2]. Escobedo proved that the Kompaneets equation develops singularities at zero energy [5]. A similar phenomenon holds for the superlinear drift equation [2. If the initial mass is large enough, the model of Kaniadakis and Quarati also admits solutions which blow up in finite time [19].

In contrast to the above mentioned models, the PDE in (1.1) is a pure diffusion equation, not explicitly containing lower-order drift terms. On the other hand, the diffusion mechanism in (1.1) is of fourth order, highly nonlinear, and it degenerates both at $x=0$ and near points where $u=0$. Mathematical challenges thus do not only result from the lack of comparison principles, but moreover also from the fact that standard parabolic regularity does not apply. Based on the construction of a family of approximate problems and weighted gradient estimates, we have proved the local-in-time existence of mass-conserving continuous weak solutions [13].

The purpose of the present paper is to go one step further and reveal a striking qualitative feature of (1.1): Namely, we shall see that even the strong simplification (1.1) of the original BoltzmannNordheim equation possesses the ability to spontaneously generate singularities. To this end, we first refine the existence theory by proving the existence of local-in-time entropy solutions to (1.1) which, as compared to the continuous weak solution constructed in [13], possess some additional properties; 
in particular, our entropy solutions will satisfy the entropy inequality (1.2) below.

Based on this inequality and some further regularity information thereby implied, we shall be able to show that if at the initial time, the mass is concentrated to a sufficient extent near the zero energy level $x=0$, then this entropy solution must cease to exist within finite time. On the other hand, from our approach to local existence, as developed in [13], we know that solutions can be extended in time as long as their norm with respect to $L^{\infty}(\Omega)$ remains bounded; consequently, any such nonglobal entropy solution must blow up in $L^{\infty}(\Omega)$. Our precise requirements quantifying the above concentration condition will be shown to be conveniently mild: We shall see that the set of all such blow-up enforcing initial data $u_{0}$ is actually dense in the set of all admissible initial conditions. This seems essentially optimal in light of the observation that all nonnegative constants trivially solve (1.1), and that hence in particular there cannot exist any critical mass level above which all solutions must blow up.

In order to precisely state these results, let us introduce some notation. For $\gamma \in \mathbb{R}$, we define the weighted Sobolev space

$$
W_{\gamma}^{1,2}(\Omega):=\left\{v \in W_{\mathrm{loc}}^{1,2}(\Omega) \mid\|v\|_{W_{\gamma}^{1,2}(\Omega)}^{2} \equiv\|v\|_{L^{2}(\Omega)}^{2}+\left\|x^{\frac{\gamma}{2}} v_{x}\right\|_{L^{2}(\Omega)}^{2}<\infty\right\}
$$

which for $\gamma<1$ satisfies $W_{\gamma}^{1,2}(\Omega) \hookrightarrow C^{0, \theta}(\bar{\Omega})$, where $\theta=\min \left\{\frac{1}{2}, \frac{1-\gamma}{2}\right\}$ [13]. We denote by $\chi_{Q}$ the characteristic function on the set $Q \subset \mathbb{R}^{n}$. For $T>0$, the space $C^{4,1}(\bar{\Omega} \times(0, T))$ consists of all functions $u$ such that $\partial u / \partial t$ and $\partial^{\alpha} u / \partial x^{\alpha}$ are continuous in $\bar{\Omega} \times(0, T)$ for all $0 \leq \alpha \leq 4$. Furthermore, for any (not necessarily open) subset $Q \subset \mathbb{R}^{n}, C_{0}^{\infty}(Q)$ is the space of all functions $u \in C^{\infty}(Q)$ such that $\operatorname{supp}(u) \subset Q$ is compact.

First, we prove the local-in-time existence of entropy solutions. We call $u$ an entropy solution to (1.1) in $\Omega \times(0, T)$ if $u$ is continuous, smooth on $\{u>0\}$, it satisfies certain weighted integrability conditions, it solves (1.1) in the weak sense, and the entropy inequality

$$
-\int_{\Omega} x^{\beta} \ln u(x, t) d x+\int_{0}^{t} \int_{\Omega} \chi_{\{u>0\}} x^{\alpha} u^{n-4}\left(u u_{x x}-2 u_{x}^{2}\right)^{2} d x d t \leq-\int_{\Omega} x^{\beta} \ln u_{0}(x) d x
$$

holds for all $t \in(0, T)$. We refer to Definition 3.2 below for the precise formulation.

Theorem 1.1 (Local existence of entropy solutions) Let $n \in\left(n_{*}, 3\right)$, where $n_{*}=1.5361 \ldots$ is the unique positive root of the polynomial $n \mapsto n^{3}+5 n^{2}+16 n-40$. Let $\alpha \in(3, \infty), \beta \in\left(-1, \frac{\alpha-n-3}{n}\right]$ and $\gamma \in(5-\alpha+\beta, 1)$, and let $u_{0} \in W_{\gamma}^{1,2}(\Omega)$ be a nonnegative function satisfying

$$
\int_{\Omega} x^{\beta} \ln u_{0}(x) d x>-\infty
$$

Then there exists $T_{\max } \in(0, \infty]$ such that (1.1) possesses at least one entropy solution $u$ in $\Omega \times\left(0, T_{\max }\right)$ in the sense of Definition 3.2 satisfying the following alternative:

$$
\text { If } T_{\max }<\infty \quad \text { then } \quad \limsup _{t \nearrow T_{\max }}\|u(\cdot, t)\|_{L^{\infty}(\Omega)}=\infty .
$$


The idea of the proof is to consider, as in [13], a family of approximate equations

$$
u_{t}=(x+\varepsilon)^{-\beta}\left(-g_{\varepsilon}(x) u^{n} u_{x x}+2 g_{\varepsilon}(x) u^{n-1} u_{x}^{2}\right)_{x x} \quad \text { in } \Omega, t>0,
$$

together with the boundary conditions in (1.1) and a family of approximate initial conditions, where $\varepsilon>0$ and $g_{\varepsilon}(x)$ approximates $x^{\alpha}$ but vanishes on the boundary. The latter condition ensures that the approximate flux $J:=-g_{\varepsilon}(x) u^{n} u_{x x}+2 g_{\varepsilon}(x) u^{n-1} u_{x}^{2}$ vanishes on the boundary as well. It is shown in 13 that on some time interval conveniently small but independent of $\varepsilon$, there exists a positive classical solution $u_{\varepsilon}$ to this aproximate problem, and that there exists a sequence of such solutions $u_{\varepsilon}$ converging to a continuous weak solution to (1.1) as $\varepsilon=\varepsilon_{j} \searrow 0$. It will turn out that, thanks to assumption (1.3), this solution actually is an entropy solution and, in particular, satisfies (1.2).

The following theorem is the main result of the paper. It states that there exist entropy solutions which blow up in finite time. We set $\ln _{+} z:=(\ln z)_{+}:=\max \{0, \ln z\}$ for $z>0$.

Theorem 1.2 (Finite-time blow-up of entropy solutions) Let $n \in\left(n_{*}, 3\right)$, where $n_{*}$ is as in Theorem 1.1, $\alpha \in(n+4, \infty)$, and $\beta \in\left(\frac{\alpha-n-4}{n+1}, \frac{\alpha-n-3}{n}\right]$. Then for all $B>0, D>0, T>0$, and each $\kappa>0$ fulfilling

$$
\kappa<\min \left\{\frac{\alpha-3}{2}, \alpha-n-4, \beta+1, \frac{-\alpha+(n+1) \beta+n+4}{n}\right\},
$$

there exists $M=M(B, D, T, \kappa)>0$ such that if $u_{0} \in C^{0}(\bar{\Omega})$ is a nonnegative function satisfying

$$
\int_{\Omega} x^{\beta} u_{0}(x) d x \leq B \quad \text { and } \quad \int_{\Omega} x^{\beta} \ln _{+} \frac{1}{u_{0}(x)} d x \leq D,
$$

but

$$
\int_{\Omega} x^{\beta-\kappa} u_{0}(x) d x \geq M
$$

then (1.1) does not possess any entropy solution in $\Omega \times(0, T)$. If additionally $u_{0} \in W_{\gamma}^{1,2}(\Omega)$ for some $\gamma \in(5-\alpha+\beta, 1)$, then the entropy solution $u$ to (1.1), as constructed in Theorem 1.1, blows up before time $T>0$; that is, in this case we have $T_{\max }<T$ and

$$
\limsup _{t \nearrow T_{\max }}\|u(\cdot, t)\|_{L^{\infty}(\Omega)}=\infty .
$$

Note that the above conditions on the parameters include the physically relevant values $n=2, \alpha=\frac{13}{2}$, and $\beta=\frac{1}{2}$. We do not pursue here the mathematical question in how far the respective ranges of $n$, $\alpha$, and $\beta$ in Theorem 1.2 are optimal.

To establish the latter blow-up result, we will firstly exploit the entropy inequality (1.2) satisfied by the entropy solution to (1.1) in order to prove some additional regularity properties in weighted Sobolev spaces. The main step then consists in deriving an integral inequality for the generalized moment functional $y(t)=\int_{\Omega} x^{\beta-\kappa} u(x, t) d x$, which will have the form

$$
y(t) \geq \int_{0}^{\frac{L}{4}} x^{\beta-\kappa} u_{0}(x) d x-C_{1}(1+T)+C_{2} \int_{0}^{t}\left(y(s)-C_{3}\right)_{+}^{n+1} d s, \quad t \in(0, T) .
$$


Here, $C_{1}, C_{2}$, and $C_{3}$ denote certain positive constants which depend on $u_{0}$ only through its mass $\int_{\Omega} x^{\beta} u_{0}(x) d x$ and the integral $\int_{\Omega} x^{\beta} \ln _{+} \frac{1}{u_{0}(x)} d x$. The proof of (1.9) uses several integrations by parts; in the considered generalized solution framework, these require additional justification which can be achieved using the asserted regularity properties enforced by (1.2). By a nonlinear Grønwall lemma, we finally conclude from (1.9) that for sufficiently large $M>0$ in Theorem 1.2, the entropy solution ceases to exist in finite time, and that hence (1.8) holds.

We expect that, under the conditions of Theorem 1.2, the entropy solution blows up at zero energy $x=0$. This conjecture is physically reasonable and investigated by formal asymptotic arguments in [11. We believe, but cannot prove rigorously here, that the validity of (1.2) provides sufficient regularity for solutions away from the origin $x=0$ so as to prevent blow-up at any $x>0$.

One may ask the question how many initial functions simultaneously satisfy (1.6) and (1.7). As an explicit construction will show, the set of all such blow-up enforcing initial data is actually large in the sense specified in the following proposition.

Proposition 1.3 (Density of blow-up enforcing initial data) Let $n, \alpha, \beta$, and $\kappa$ be as in Theorem 1.2. Then for any $p \in\left(0, \frac{1}{\beta+1-\kappa}\right)$ and each nonnegative $u_{0} \in C^{0}(\bar{\Omega})$ satisfying $\int_{\Omega} x^{\beta} \ln u_{0}(x) d x>$ $-\infty$, one can find a sequence of nonnegative functions $u_{0 k}$ such that $u_{0 k}-u_{0} \in C_{0}^{\infty}(\Omega)$ for all $k \in \mathbb{N}$ and such that $u_{0 k} \rightarrow u_{0}$ in $L^{p}(\Omega)$ as $k \rightarrow \infty$ and

$$
\int_{\Omega} x^{\beta} u_{0 k}(x) d x \rightarrow \int_{\Omega} x^{\beta} u_{0}(x) d x \quad \text { as } k \rightarrow \infty
$$

as well as

$$
\int_{\Omega} x^{\beta} \ln _{+} \frac{1}{u_{0 k}(x)} d x \rightarrow \int_{\Omega} x^{\beta} \ln _{+} \frac{1}{u_{0}(x)} d x \quad \text { as } k \rightarrow \infty
$$

but such that

$$
\int_{\Omega} x^{\beta-\kappa} u_{0 k}(x) d x \rightarrow+\infty \quad \text { as } k \rightarrow \infty .
$$

In order to highlight a peculiarity in the approach toward blow-up pursued in this paper, let us recall that tracking the time evolution of moments such as in (1.9), or of related linear functionals of solutions, has a long history in the blow-up analysis of parabolic equations. In fact, approaches of this type have widely been applied to reveal singularity formation driven by superlinear forces or nonlinear drift terms, both in second-order as well as in higher-order diffusion equations [4, 8, 15, 17]. In contrast to this, arguments based on dissipation through the exploitation of entropy- (or energy-) like inequalities usually can be applied to detect blow-up solutions only when the respective entropy functional is unbounded from below and hence can diverge to $-\infty$; examples for such reasonings again include reaction-diffusion equations [17, 18, and long-wave unstable thin-film equations [1, but also some more complex parabolic systems such as the Keller-Segel system [20] as well as dispersive equations such as nonlinear wave and Schrödinger equations [9, 12, 17.

Now in the present context, the entropy functional $-\int_{\Omega} x^{\beta} \ln u(x, t) d x$ is a priori bounded from below along trajectories because of mass conservation; accordingly, the associated entropy inequality (1.2) might be expected to enforce regularity, boundedness and possibly even stabilization of solutions (cf. e.g. [3]), rather than support their explosion. Surprisingly, precisely this additional regularity implied by (1.2) constitutes an indispensable cornerstone in our blow-up analysis by providing appropriate estimates which finally allow for the derivation of the inequality (1.9) for the considered 
moment functional $y(t)$.

The paper is organized as follows. In Section 2, we prove some interpolation inequalities in weighted Sobolev spaces, needed in the following sections. The local-in-time existence of entropy solutions, as formulated in Theorem 1.1, is proved in Section 3. Section 4 is concerned with the proof of the integral inequality (1.9) and of Theorem 1.2 and Proposition 1.3. The construction of approximate smooth initial data, needed in the proof of Theorem 1.2, is shown in the appendix.

\section{Some interpolation inequalities}

Let us first show some interpolation inequalities used in the sequel (cf. e.g. Lemma 4.4). A common ingredient is the following basic inequality.

Lemma 2.1 Let $\beta \in \mathbb{R}$. Then for all nonnegative $u \in L_{\text {loc }}^{1}(\Omega)$ there exists $x_{0} \in\left(\frac{L}{2}, L\right)$ such that

$$
u\left(x_{0}\right) \leq C \int_{\Omega} x^{\beta} u(x) d x
$$

holds with $C:=\left(\int_{\frac{L}{2}}^{L} x^{\beta} d x\right)^{-1}$.

Proof. This is immediately clear, for assuming $u(x)>C \int_{\Omega} x^{\beta} u(x) d x$ to hold in $\left(\frac{L}{2}, L\right)$ would lead to the absurd conclusion $\int_{\Omega} x^{\beta} u(x) d x>\int_{\frac{L}{2}}^{L} x^{\beta} \cdot\left(C \int_{\Omega} y^{\beta} u(y) d y\right) d x=\int_{\Omega} y^{\beta} u(y) d y$.

The assumptions required in the following lemma explain the second to last and especially the last restriction on $\kappa$ made in Theorem 1.2 ,

Lemma 2.2 Let $n>-1, \alpha \in \mathbb{R}, \beta \in \mathbb{R}$, and $\kappa \in \mathbb{R}$ be such that

$$
\kappa<\min \left\{\alpha-3, \beta+1, \frac{-\alpha+(n+1) \beta+n+4}{n}\right\} .
$$

Then there exists $C>0$ such that if $u \in C^{0}(\bar{\Omega})$ is nonnegative and $u \in C^{1}(\{u>0\})$, we have

$$
\int_{\Omega} x^{\beta-\kappa} u(x) d x \leq C \int_{\Omega} x^{\beta} u(x) d x+C\left(\int_{\Omega} \chi_{\{u>0\}} x^{\alpha-\kappa-2} u^{n-1} u_{x}^{2} d x\right)^{\frac{1}{n+1}} .
$$

Proof. Assuming without loss of generality that $B:=\int_{\Omega} x^{\beta} u(x) d x$ and the second integral on the right-hand side of (2.3) are both finite, from Lemma 2.1 we obtain $c_{1}>0$ and $x_{0} \in\left(\frac{L}{2}, L\right)$ such that $u\left(x_{0}\right) \leq c_{1} B$. Now, let $x \in\left(0, \frac{L}{2}\right)$ be such that $u(x)>0$, and let $x_{1}:=\sup \left\{\tilde{x} \in\left(x, x_{0}\right) \mid u>\right.$ 0 in $[x, \tilde{x}]\}$. Then either $x_{1}<x_{0}$, which implies that $u\left(x_{1}\right)=0 \leq c_{1} B$, or $x_{1}=x_{0}$, meaning that $u\left(x_{1}\right)=u\left(x_{0}\right) \leq c_{1} B$. Since clearly $u>0$ in $\left[x, x_{1}\right)$, our assumptions assert that $u^{\frac{n+1}{2}}$ belongs to $C^{0}\left(\left[x, x_{1}\right]\right) \cap C^{1}\left(\left(x, x_{1}\right)\right)$, so that by the Cauchy-Schwarz inequality, we can estimate

$$
\begin{aligned}
u^{\frac{n+1}{2}}(x) & =u^{\frac{n+1}{2}}\left(x_{1}\right)+\int_{x_{1}}^{x}\left(u^{\frac{n+1}{2}}\right)_{x}(y) d y \\
& \leq\left(c_{1} B\right)^{\frac{n+1}{2}}+\left(\int_{\Omega} \chi_{\{u>0\}} y^{\alpha-\kappa-2}\left(u^{\frac{n+1}{2}}\right)_{x}^{2}(y) d y\right)^{\frac{1}{2}} \cdot\left|\int_{x_{1}}^{x} y^{-\alpha-\kappa+2} d y\right|^{\frac{1}{2}} .
\end{aligned}
$$


As $\alpha-\kappa-3>0$, we have

$$
\left|\int_{x_{1}}^{x} y^{-\alpha+\kappa+2} d y\right|=\frac{1}{\alpha-\kappa-3}\left(x^{-\alpha+\kappa+3}-x_{1}^{-\alpha+\kappa-3}\right) \leq \frac{x^{-\alpha+\kappa+3}}{\alpha-\kappa-3},
$$

whence using that $(a+b)^{m} \leq 2^{m}\left(a^{m}+b^{m}\right)$ for all $a \geq 0, b \geq 0$, and $m>0$ we obtain

$$
\begin{aligned}
u(x) & \leq\left\{\left(c_{1} B\right)^{\frac{n+1}{2}}+\frac{n+1}{2 \sqrt{\alpha-\kappa-3}} \cdot x^{\frac{-\alpha+\kappa+3}{2}} \cdot\left(\int_{\Omega} \chi_{\{u>0\}} y^{\alpha-\kappa-2} u^{n-1} u_{x}^{2} d x\right)^{\frac{1}{2}}\right\}^{\frac{2}{n+1}} \\
& \leq 2^{\frac{2}{n+1}} c_{1} B+c_{2} x^{\frac{-\alpha+\kappa+3}{n+1}} \cdot\left(\int_{\Omega} \chi_{\{u>0\}} y^{\alpha-\kappa-2} u^{n-1} u_{x}^{2} d x\right)^{\frac{1}{n+1}}
\end{aligned}
$$

with $c_{2}:=2^{\frac{2}{n+1}} \cdot \frac{n+1}{2 \sqrt{\alpha-\kappa-3}}$.

We now multiply this by $x^{\beta-\kappa}$ and integrate over $\left(0, \frac{L}{2}\right)$. Noting that $\kappa<\beta+1$ implies that $c_{3}:=$ $\int_{0}^{\frac{L}{2}} x^{\beta-\kappa} d x$ is finite, whereas (2.2) ensures that also $c_{4}:=\int_{0}^{L} x^{\beta-\kappa+\frac{-\alpha+\kappa+3}{n+1}} d x$ is finite, from (2.4) we thereby derive the inequality

$$
\int_{0}^{\frac{L}{2}} x^{\beta-\kappa} u(x) d x \leq 2^{\frac{2}{n+1}} c_{1} c_{3} B+c_{2} c_{4}\left(\int_{\Omega} \chi_{\{u>0\}} x^{\alpha-\kappa-2} u^{n-1} u_{x}^{2} d x\right)^{\frac{1}{n+1}} .
$$

Combined with the trivial estimate

$$
\int_{\frac{L}{2}}^{L} x^{\beta-\kappa} u(x) d x \leq \max \left\{\left(\frac{L}{2}\right)^{-\kappa}, L^{-\kappa}\right\} \cdot \int_{\Omega} x^{\beta} u(x) d x,
$$

this proves (2.3).

Lemma 2.3 Let $n>1, \alpha>3$, and $\beta>-1$ be such that

$$
\beta \leq \frac{\alpha-n-3}{n}
$$

Then for all $\eta>0$, there exists $C(\eta)>0$ such that for any nonnegative $u \in C^{0}(\bar{\Omega})$ with $u \in C^{1}(\{u>0\}$ we have

$$
\int_{\Omega} x^{\alpha-4} u^{n}(x) d x \leq \eta \int_{\Omega} x^{\alpha} u^{n-4} u_{x}^{4} d x+C(\eta)\left(\int_{\Omega} x^{\beta} u(x) d x\right)^{n}
$$

Proof. $\quad$ Proceeding as in Lemma 2.2, by Lemma 2.1, we find $x_{0} \in\left(\frac{L}{2}, L\right)$ and $c_{1}>0$ such that $u\left(x_{0}\right) \leq c_{1} B$ holds with $B:=\int_{\Omega} x^{\beta} u(x) d x$. For fixed $x \in \Omega \cap\{u>0\}$, we then let

$$
x_{1}:= \begin{cases}\sup \left\{\tilde{x} \in\left(x, x_{0}\right) \mid u>0 \text { in }[x, \tilde{x}]\right\} & \text { if } x<x_{0}, \\ \inf \left\{\tilde{x} \in\left[x_{0}, x\right] \mid u>0 \text { in }[\tilde{x}, x]\right\} & \text { if } x \geq x_{0},\end{cases}
$$


and then obtain again $u\left(x_{1}\right) \leq c_{1} B$. Furthermore, if $x \neq x_{0}$ then $u^{\frac{n}{4}}$ is continuous in $\overline{\left\langle x, x_{1}\right\rangle}$ and moreover positive and hence continuously differentiable in $\left\langle x, x_{1}\right\rangle$, where $\left\langle x, x_{1}\right\rangle:=\left(x, x_{1}\right)$ if $x<x_{1}$ and $\left\langle x, x_{1}\right\rangle:=\left(x_{1}, x\right)$ if $x>x_{1}$. Therefore, using the Hölder inequality, we see that

$$
\begin{aligned}
u^{\frac{n}{4}}(x) & =u^{\frac{n}{4}}\left(x_{1}\right)+\int_{x_{1}}^{x}\left(u^{\frac{n}{4}}\right)_{x}(y) d y \\
& \leq\left(c_{1} B\right)^{\frac{n}{4}}+\left(\int_{\Omega} \chi_{\{u>0\}} y^{\alpha}\left(u^{\frac{n}{4}}\right)_{x}^{4}(y) d y\right)^{\frac{1}{4}}\left|\int_{x_{1}}^{x} y^{-\frac{\alpha}{3}} d y\right|^{\frac{3}{4}},
\end{aligned}
$$

where regardless of the position of $x_{1}$ relative to $x$ we have

$$
\left|\int_{x_{1}}^{x} y^{-\frac{\alpha}{3}} d y\right|=\left|\frac{3}{\alpha-3}\left(x^{\frac{3-\alpha}{3}}-x_{1}^{\frac{3-\alpha}{3}}\right)\right| \leq \frac{3 \cdot 2^{\frac{\alpha-3}{3}}}{\alpha-3} \cdot x^{\frac{3-\alpha}{3}}
$$

due to the fact that $\alpha>3$ and the obvious inequality $x_{1} \geq \frac{x}{2}$ (since if $x<x_{0}$, we have $x_{1}>x>\frac{x}{2}$ and otherwise, $x_{1} \geq x_{0}>\frac{L}{2} \geq \frac{x}{2}$ ). Consequently, from (2.7) we conclude that

$$
\begin{aligned}
x^{\alpha-\beta-4} u^{n-1}(x) \leq 2^{\frac{4(n-1)}{n}}\{ & \left(c_{1} B\right)^{n-1} x^{\alpha-\beta-4} \\
& \left.+\left(\frac{3 \cdot 2^{\frac{\alpha-3}{3}}}{\alpha-3}\right)^{\frac{3(n-1)}{n}} \cdot x^{\alpha-\beta-4+\frac{(n-1)(3-\alpha)}{n}}\left(\int_{\Omega} y^{\alpha} \chi_{\{u>0\}}\left(u^{\frac{n}{4}}\right)_{x}^{4}(y) d y\right)^{\frac{n-1}{n}}\right\}
\end{aligned}
$$

holds for all $x \in \Omega$, so that since $\alpha-\beta-4>\alpha-\beta-4+\frac{(n-1)(3-\alpha)}{n} \geq 0$ by (2.5), we find $c_{2}>0$ fulfilling

$$
x^{\alpha-\beta-4} u^{n-1}(x) \leq c_{2} B^{n-1}+c_{2}\left(\int_{\Omega} \chi_{\{u>0\}} y^{\alpha} u^{n-4} u_{x}^{4} d y\right)^{\frac{n-1}{n}} \quad \text { for all } x \in \Omega .
$$

As

$$
\int_{\Omega} x^{\alpha-4} u^{n}(x) d x \leq \sup _{x \in \Omega}\left(x^{\alpha-\beta-4} u^{n-1}(x)\right) \cdot \int_{\Omega} x^{\beta} u(x) d x
$$

an application of Young's inequality easily leads to (2.6).

Lemma 2.4 Let $n>0, p>1$, and $\Omega_{0} \subset \mathbb{R}$ be a bounded interval. Then there exists $C>0$ such that for each nonnegative $u \in C^{0}\left(\bar{\Omega}_{0}\right)$ with $u \in C^{1}(\{u>0\})$, we have

$$
\int_{\Omega_{0}} u^{p} d x \leq C\left\{\left(\int_{\Omega_{0}} u d x\right)^{\frac{n+3 p}{n+3}}\left(\int_{\Omega_{0}} \chi_{\{u>0\}} u^{n-4} u_{x}^{4} d x\right)^{\frac{p-1}{n+3}}+\left(\int_{\Omega_{0}} u d x\right)^{p}\right\} .
$$

Proof. We only need to consider the case

$$
\int_{\Omega_{0}} \chi_{\{u>0\}} u^{n-4} u_{x}^{4} d x<\infty
$$

in which we use a cut-off argument: With a fixed $\zeta \in C^{\infty}(\mathbb{R})$ such that $\zeta \equiv 0$ in $(-\infty, 1], \zeta \equiv 1$ in $[2, \infty)$ and $0 \leq \zeta^{\prime} \leq 2$ on $\mathbb{R}$, for $\delta>0$ and $s \geq 0$, we introduce a regularization $\chi_{\delta}(s)$ of $\chi_{\{s>0\}}$ 
by defining $\chi_{\delta}(s):=\zeta\left(\frac{s}{\delta}\right)$. We then may apply the Gagliardo-Nirenberg inequality to each of the functions $\chi_{\delta}(u) u^{\frac{n}{4}}, \delta>0$, so as to obtain $c_{1}>0$ fulfilling

$$
\begin{aligned}
\int_{\Omega_{0}} \chi_{\delta}^{\frac{4 p}{n}}(u) d x \cdot u^{p} & =\left\|\chi_{\delta}(u) u^{\frac{n}{4}}\right\|_{L^{\frac{4 p}{n}}\left(\Omega_{0}\right)}^{\frac{4 p}{n}} \\
& \leq c_{1}\left\|\left(\chi_{\delta}(u) u^{\frac{n}{4}}\right)_{x}\right\|_{L^{4}\left(\Omega_{0}\right)}^{\frac{4(p-1)}{n+3}}\left\|\chi_{\delta}(u) u^{\frac{n}{4}}\right\|_{L^{\frac{4}{n}\left(\Omega_{0}\right)}}^{\frac{4(n+3 p)}{n(n+3)}}+c_{1}\left\|\chi_{\delta}(u) u^{\frac{n}{4}}\right\|_{L^{\frac{4}{n}}\left(\Omega_{0}\right)}^{\frac{4 p}{n}}
\end{aligned}
$$

Here, with some $c_{2}>0$ we have

$$
\left\|\left(\chi_{\delta}(u) u^{\frac{n}{4}}\right)_{x}\right\|_{L^{4}\left(\Omega_{0}\right)}^{4} \leq c_{2}\left(\int_{\Omega_{0}} \chi_{\delta}^{\prime 4}(u) u^{n} u_{x}^{4} d x+\int_{\Omega_{0}} \chi_{\delta}^{4}(u) u^{n-4} u_{x}^{4} d x\right)
$$

and recalling that $\chi_{\delta}^{\prime} \equiv 0$ outside of the interval $\{\delta<u<2 \delta\}$ and $\left\|\chi_{\delta}^{\prime}\right\|_{L^{\infty}(\mathbb{R})} \leq \frac{2}{\delta}$, we may use the dominated convergence theorem along with (2.9) to infer that

$$
\int_{\Omega_{0}} \chi_{\delta}^{\prime 4}(u) u^{n} u_{x}^{4} d x \rightarrow 0 \quad \text { as } \delta \rightarrow 0
$$

because

$$
\chi_{\delta}^{\prime 4}(u) u^{n} u_{x}^{4} \leq\left(\frac{2}{\delta}\right)^{4}(2 \delta)^{4} u^{n-4} u_{x}^{4} \quad \text { in } \Omega_{0} .
$$

Using that $\chi_{\delta}(u) \nearrow \chi_{\{u>0\}}$ in $\Omega_{0}$ as $\delta \searrow 0$, from (2.10) and Beppo Levi's theorem we thus readily obtain (2.8).

\section{Local existence and extensibility of entropy solutions}

In this section, we prove the local existence of entropy solutions.

\subsection{Continuous weak solutions and entropy solutions}

We first recall the definition of continuous weak solutions from [13].

Definition 3.1 (Continuous weak solution) Let $n, \alpha, \beta \in \mathbb{R}$, and $T>0$, and suppose that $u_{0} \in$ $C^{0}(\bar{\Omega})$ is nonnegative. Then by a continuous weak solution of (1.1) in $\Omega \times(0, T)$ we mean a nonnegative function $u \in C^{0}(\bar{\Omega} \times[0, T))$ with the properties $u \in C^{4,1}(((0, L] \times(0, T)) \cap\{u>0\})$ as well as

$$
\chi_{\{u>0\}} x^{\alpha} u^{n} u_{x x} \in L_{l o c}^{1}(\bar{\Omega} \times[0, T)) \quad \text { and } \quad \chi_{\{u>0\}} x^{\alpha} u^{n-1} u_{x}^{2} \in L_{l o c}^{1}(\bar{\Omega} \times[0, T)),
$$

for which $u(\cdot, t)$ is differentiable with repect to $x$ at the point $x=L$ for a.e. $t \in(0, T)$ with

$$
u_{x}(L, t)=0 \quad \text { for a.e. } t \in(0, T),
$$

and which satisfies the integral identity

$$
-\int_{0}^{T} \int_{\Omega} x^{\beta} u \phi_{t} d x d t-\int_{\Omega} x^{\beta} u_{0} \phi(\cdot, 0) d x=\int_{0}^{T} \int_{\Omega} \chi_{\{u>0\}}\left[-x^{\alpha} u^{n} u_{x x}+2 x^{\alpha} u^{n-1} u_{x}^{2}\right] \phi_{x x} d x d t
$$

for all $\phi \in C_{0}^{\infty}(\bar{\Omega} \times[0, T))$ fulfilling $\phi_{x}(L, t)=0$ for all $t \in(0, T)$. 
The existence of local-in-time continuous weak solutions to (1.1) for certain parameters $(n, \alpha, \beta)$ was proved in 13. Any such solution satisfies the natural mass conservation property associated with (1.1).

Lemma 3.1 (Mass conservation) Let $n>0, \alpha>0, \beta>-1, T>0$, and $u_{0} \in C^{0}(\bar{\Omega})$ be nonnegative. Then any continuous weak solution $u$ of (1.1) in $\Omega \times(0, T)$ satisfies the identity

$$
\int_{\Omega} x^{\beta} u(x, t) d x=\int_{\Omega} x^{\beta} u_{0}(x) d x \quad \text { for all } t \in(0, T) .
$$

Proof. We fix a nonincreasing function $\zeta \in C^{\infty}(\mathbb{R})$ such that $\zeta \equiv 1$ in $(-\infty, 0]$, and $\zeta \equiv 0$ in $[1, \infty)$. For given $t_{0} \in(0, T)$, we let $\xi_{\delta}(t):=\zeta\left(\frac{t-t_{0}}{\delta}\right)$ for $t \in[0, T]$ and $\delta \in\left(0, T-t_{0}\right)$. Then $\phi(x, t):=\xi_{\delta}(t)$ defines a function $\phi \in C_{0}^{\infty}(\bar{\Omega} \times[0, T))$, and inserting $\phi$ into (3.3) yields

$$
-\frac{1}{\delta} \int_{t_{0}}^{t_{0}+\delta} \int_{\Omega} x^{\beta} u(x, t) \zeta^{\prime}\left(\frac{t-t_{0}}{\delta}\right) d x d t=\int_{\Omega} x^{\beta} u_{0}(x) d x
$$

for any such $\delta$. Taking $\delta \searrow 0$ and using the continuity of $u$, we readily end up with (3.4).

Based on the above definition, a natural additional requirement leads to the following solution concept.

Definition 3.2 (Entropy solution) Let $n>0, \alpha>0$, and $\beta \in \mathbb{R}$, and suppose that $u_{0} \in C^{0}(\bar{\Omega})$ is nonnegative and satisfies $\int_{\Omega} x^{\beta} \ln u_{0}(x) d x>-\infty$. Then given $T>0$, we call a function $u$ an entropy solution of (1.1) in $\Omega \times(0, T)$ if $u$ is a continuous weak solution in $\Omega \times(0, T)$, if moreover

$$
\begin{aligned}
& \chi_{\{u>0\}} x^{\alpha} u^{n-2} u_{x x}^{2} \in L_{l o c}^{1}(\bar{\Omega} \times[0, T)) \quad \text { and } \\
& \chi_{\{u>0\}} x^{\alpha} u^{n-4} u_{x}^{4} \in L_{l o c}^{1}(\bar{\Omega} \times[0, T)),
\end{aligned}
$$

and if the entropy inequality

$$
\begin{aligned}
-\int_{\Omega} x^{\beta} \ln u(x, t) d x+ & \int_{0}^{t} \int_{\Omega} \chi_{\{u>0\}} x^{\alpha} u^{n-2} u_{x x}^{2} d x d t+4 \int_{0}^{t} \int_{\Omega} \chi_{\{u>0\}} x^{\alpha} u^{n-4} u_{x}^{4} d x d t \\
& -4 \int_{0}^{t} \int_{\Omega} \chi_{\{u>0\}} x^{\alpha} u^{n-3} u_{x}^{2} u_{x x} d x d t \\
\leq & -\int_{\Omega} x^{\beta} \ln u_{0}(x) d x
\end{aligned}
$$

is valid for all $t \in(0, T)$.

Remark. It is clear from Young's inequality that (3.5) asserts that the integrand in the last summand on the left-hand side of $(\underline{3.6})$ belongs to $L_{l o c}^{1}(\bar{\Omega} \times[0, T))$. Moreover, for any continuous function $u$ in $\bar{\Omega} \times[0, T)$, the first term on the left-hand side of (3.6) is well-defined with values a priori possibly lying in $(-\infty, \infty]$; in view of the assumption $\int_{\Omega} x^{\beta} \ln u_{0}(x) d x>-\infty$, however, (3.6) in particular says that this term is finite for all $t \in(0, T)$ if $u$ is an entropy solution in $\Omega \times(0, T)$. The sum of the second, third and fourth integrals on the right-hand side of (3.6) can be written as one single integral with its integrand being a square,

$$
\chi_{\{u>0\}}\left(u^{n-2} u_{x x}^{2}+4 u^{n-4} u_{x}^{4}-4 u^{n-3} u_{x}^{2} u_{x x}\right)=\chi_{\{u>0\}} u^{n-4}\left(u u_{x x}-2 u_{x}^{2}\right)^{2} .
$$

This justifies the notion of "entropy" for the integral $-\int_{\Omega} x^{\beta} \ln u(x, t) d x$ used in, e.g., [3]. 


\subsection{A family of approximate problems}

Following the approach in [13], we will construct entropy solutions of (1.1) as limits of solutions to the regularized problems

$$
\left\{\begin{array}{l}
u_{t}=\frac{1}{(x+\varepsilon)^{\beta}} \cdot\left\{-g_{\varepsilon}(x) u^{n} u_{x x}+2 g_{\varepsilon}(x) u^{n-1} u_{x x}\right\}_{x x}, \quad x \in \Omega, t>0 \\
u_{x}=u_{x x x}=0, \quad x \in \partial \Omega, t>0
\end{array}\right.
$$

with appropriate initial data, where $0<\varepsilon<\varepsilon_{0}:=\min \left\{1, \sqrt{\frac{L}{2}}\right\}$ and $g_{\varepsilon} \in C^{\infty}([0, L])$ is the positive function given by $g_{\varepsilon}:=z_{\varepsilon}^{\alpha}$ with

$$
z_{\varepsilon}(x):=\varepsilon+\int_{0}^{x} \zeta_{\varepsilon}(y) d y, \quad x \in[0, L]
$$

for some fixed $\zeta_{\varepsilon} \in C_{0}^{\infty}((0, L))$ fulfilling $0 \leq \zeta_{\varepsilon} \leq 1$ in $(0, L)$ and $\zeta_{\varepsilon} \equiv 1$ in $\left(\varepsilon^{2}, L-\varepsilon^{2}\right)$. Then the fact that $g_{\varepsilon x}=0$ at both $x=0$ and $x=L$ ensures that whenever $u \in C^{4,1}(\bar{\Omega})$ is a positive classical solution of (3.7) in $\Omega \times(0, T)$ for some $T>0$, the function $J=J[u]$ defined by

$$
J(x, t):=J[u](x, t):=-g_{\varepsilon}(x) u^{n} u_{x x}+2 g_{\varepsilon}(x) u^{n-1} u_{x}^{2}, \quad x \in \bar{\Omega}, t \in(0, T),
$$

satisfies

$$
J_{x}(x, t)=0 \quad \text { for all } x \in \partial \Omega \text { and } t \in(0, T)
$$

(cf. [13, Lemma 2.2]). Moreover, we shall need the following approximation properties of $\left(g_{\varepsilon}\right)_{\varepsilon \in\left(0, \varepsilon_{0}\right)}$.

Lemma 3.2 Let $\alpha>0$. Then

$$
g_{\varepsilon}(x) \rightarrow x^{\alpha} \quad \text { and } \quad g_{\varepsilon x}(x) \rightarrow \alpha x^{\alpha-1} \quad \text { locally uniformly with respect to } x \in \Omega
$$

as $\varepsilon \searrow 0$. Furthermore, there exists $C>0$ such that

$$
\frac{g_{\varepsilon x}^{4}(x)}{g_{\varepsilon}^{3}(x)} \leq C(x+\varepsilon)^{\alpha-4} \quad \text { for all } x \in \Omega \text { and each } \varepsilon \in\left(0, \varepsilon_{0}\right) .
$$

Proof. The properties in (3.11) are immediate from the definition of $g_{\varepsilon}$. To verify (3.12), we only need to observe that there exist $c_{1}>0$ and $c_{2}>0$ such that whenever $\varepsilon \in\left(0, \varepsilon_{0}\right)$,

$$
c_{1}(x+\varepsilon)^{\alpha} \leq g_{\varepsilon}(x) \leq(x+\varepsilon)^{\alpha} \quad \text { for all } x \in \Omega
$$

and

$$
0 \leq g_{\varepsilon x}(x) \leq c_{2}(x+\varepsilon)^{\alpha-1} \quad \text { for all } x \in \Omega
$$

(see also [13, Lemma 2.1]). 


\subsection{A criterion ensuring that a continuous weak solution is an entropy solution}

In this section we shall make sure that any continuous weak solution already is an entropy solution if it can be suitably approximated by solutions of (3.7). In deriving this in Lemma 3.4 below, we shall make use of the following statement on integration by parts for possibly nonsmooth functions.

Lemma 3.3 (Integration by parts I) Let $n>1, \alpha>3, \beta \in\left(-1, \frac{\alpha-n-3}{n}\right)$, and $T>0$. Suppose that $\left.u \in C^{0}(\bar{\Omega} \times[0, T)) \cap C^{2,0}((0, L] \times(0, T)) \cap\{u>0\}\right)$ is nonnegative and such that (3.5) holds as well as

$$
u_{x}(L, t)=0 \quad \text { for a.e. } t \in(0, T) \text { at which } u(L, t)>0 .
$$

Then

$$
\begin{aligned}
\int_{0}^{t} \int_{\Omega} \chi_{\{u>0\}} x^{\alpha} u^{n-3} u_{x}^{2} u_{x x} d x d s= & \frac{3-n}{3} \int_{0}^{t} \int_{\Omega} \chi_{\{u>0\}} x^{\alpha} u^{n-4} u_{x}^{4} d x d s \\
& -\frac{\alpha}{3} \int_{0}^{t} \int_{\Omega} \chi_{\{u>0\}} x^{\alpha-1} u^{n-3} u_{x}^{3} d x d s
\end{aligned}
$$

for all $t \in(0, T)$.

Proof. Because of possible singularities at $x=0$ and $t=0$, we need to regularize. To this end, we once more fix $\zeta \in C^{\infty}(\mathbb{R})$ such that $\zeta \equiv 0$ in $(-\infty, 1]$ and $\zeta \equiv 1$ in $[2, \infty)$ as well as $0 \leq \zeta^{\prime} \leq 2$. Moreover, for $\eta \in\left(0, \frac{L}{2}\right)$ and $\delta>0$ we let

$$
\psi_{\eta}(x):=\zeta\left(\frac{x}{\eta}\right), \quad x \in \bar{\Omega}
$$

and

$$
\chi_{\delta}(s):=\zeta\left(\frac{s}{\delta}\right), \quad s \geq 0 .
$$

The function $\psi_{\eta}$ removes the singularity at $x=0$ since $x^{\alpha} \psi_{\eta}(x)=0$ in $(0, \eta)$, and the function $\chi_{\delta}$ approximates $\chi_{\{u>0\}}$. Now, for each fixed $t \in(0, T)$, using Young's inequality and (3.5), we find that

$$
\begin{aligned}
\int_{0}^{t} \int_{\Omega} \chi_{\{u>0\}} x^{\alpha} u^{n-3} u_{x}^{2}\left|u_{x x}\right| d x d s \leq & \int_{0}^{t} \int_{\Omega} \chi_{\{u>0\}} x^{\alpha} u^{n-2} u_{x x}^{2} d x d s \\
& +\int_{0}^{t} \int_{\Omega} \chi_{\{u>0\}} x^{\alpha} u^{n-4} u_{x}^{4} d x d s<\infty,
\end{aligned}
$$

so that the dominated convergence theorem tells us that

$$
\int_{0}^{t} \int_{\Omega} \chi_{\{u>0\}} x^{\alpha} u^{n-3} u_{x}^{2} u_{x x} d x d s=\lim _{\tau \searrow 0} \lim _{\delta \searrow 0} \lim _{\eta \searrow 0} I(\tau, \eta, \delta),
$$

where we have set

$$
I(\tau, \eta, \delta):=\int_{\tau}^{t} \int_{\Omega} x^{\alpha} \psi_{\eta}(x) \chi_{\delta}(u) u^{n-3} u_{x}^{2} u_{x x} d x d s
$$


for $\tau \in(0, t), \eta \in\left(0, \frac{L}{2}\right)$, and $\delta>0$. On the other hand, an integration by parts shows that

$$
\begin{aligned}
I(\tau, \eta, \delta)= & \frac{3-n}{3} \int_{\tau}^{t} \int_{\Omega} x^{\alpha} \psi_{\eta}(x) \chi_{\delta}(u) u^{n-4} u_{x}^{4} d x d s-\frac{\alpha}{3} \int_{\tau}^{t} \int_{\Omega} x^{\alpha-1} \psi_{\eta}(x) \chi_{\delta}(u) u^{n-3} u_{x}^{3} d x d s \\
& -\frac{1}{3} \int_{\tau}^{t} \int_{\Omega} x^{\alpha} \psi_{\eta}(x) \chi_{\delta}^{\prime}(u) u^{n-3} u_{x}^{4} d x d s-\frac{1}{3} \int_{\tau}^{t} \int_{\Omega} x^{\alpha} \psi_{\eta x}(x) \chi_{\delta}(u) u^{n-3} u_{x}^{3} d x d s \\
=: & I_{1}(\tau, \eta, \delta)+I_{2}(\tau, \eta, \delta)+I_{3}(\tau, \eta, \delta)+I_{4}(\tau, \eta, \delta)
\end{aligned}
$$

for all $\tau \in(0, t), \eta \in\left(0, \frac{L}{2}\right)$ and $\delta>0$. Here, we note that the respective boundary terms vanish, because $\psi_{\eta} \equiv 0$ in $[0, \eta]$, and because for a.e. $t_{0} \in(0, t)$, we have the alternative that either $u\left(L, t_{0}\right)=0$ and hence $\chi_{\delta}\left(u\left(\cdot, t_{0}\right)\right) \equiv 0$ near $x=L$, or otherwise $u\left(L, t_{0}\right)>0$ and thus $u_{x}\left(L, t_{0}\right)=0$ according to the hypothesis (3.13). Now, taking $\eta \searrow 0$ and then $\delta \searrow 0$, we observe that $\psi_{\eta} \nearrow 1$ in $\Omega$ and $\chi_{\delta}^{\prime} \rightarrow 0$ a.e. in $(0, \infty)$, and thus we infer from Lebesgue's theorem that

$$
\lim _{\delta \searrow 0} \lim _{\eta \searrow 0} I_{3}(\tau, \eta, \delta)=0
$$

because

$$
\left|x^{\alpha} \psi_{\eta}(x) \chi_{\delta}^{\prime}(u) u^{n-3} u_{x}^{4}\right| \leq \chi_{\{u>0\}} x^{\alpha} \cdot \frac{2}{\delta} \cdot 2 \delta \cdot u^{n-4} u_{x}^{4}
$$

and the latter function belongs to $L^{1}(\Omega \times(0, t))$ according to (3.5). Similarly, we find that

$$
\left|x^{\alpha} \psi_{\eta x}(x) \chi_{\delta}(u) u^{n-3} u_{x}^{3}\right| \leq \chi_{\{u>0\}} x^{\alpha-1} \cdot 2 \eta \cdot \frac{2}{\eta} \cdot u^{n-3}\left|u_{x}\right|^{3},
$$

where thanks to our restriction $\beta \leq \frac{\alpha-n-3}{n}$, we may apply Lemma 2.3 to see that the latter expression is integrable in $\Omega \times(0, t)$, because by Young's inequality, we have

$$
\chi_{\{u>0\}} x^{\alpha-1} u^{n-3}\left|u_{x}\right|^{3} \leq \chi_{\{u>0\}} x^{\alpha} u^{n-4} u_{x}^{4}+x^{\alpha-4} u^{n},
$$

and because the latter term belongs to $L^{1}(\Omega \times(0, t))$ since $\alpha>3$. Therefore, using $\psi_{\eta x} \rightarrow 0$ a.e. in $\Omega$ as $\eta \searrow 0$, again by dominated convergence, we conclude that

$$
\lim _{\delta \searrow 0} \lim _{\eta \searrow 0} I_{4}(\tau, \eta, \delta)=0 .
$$

Since clearly the same theorem ensures that

$$
\lim _{\tau \searrow 0} \lim _{\delta \searrow 0} \lim _{\eta \searrow 0} I_{1}(\tau, \eta, \delta)=\frac{3-n}{3} \int_{0}^{t} \int_{\Omega} \chi_{\{u>0\}} x^{\alpha} u^{n-4} u_{x}^{4} d x d s
$$

and

$$
\lim _{\tau \searrow 0} \lim _{\delta \searrow 0} \lim _{\eta \searrow 0} I_{2}(\tau, \eta, \delta)=-\frac{\alpha}{3} \int_{0}^{t} \int_{\Omega} \chi_{\{u>0\}} x^{\alpha-1} u^{n-3} u_{x}^{3} d x d s,
$$

the identity (3.14) results from (3.15).

With this preparation at hand, we can indeed verify that any continuous weak solution to (1.1) which is the limit of approximate solutions to (3.7) is also an entropy solution. 
Lemma 3.4 (Continuous weak solutions yielding entropy solutions) Let $n>1, \alpha>3, \beta \in$ $\left(-1, \frac{\alpha-n-3}{n}\right)$, and $T>0$. Suppose that $u_{0} \in C^{0}(\bar{\Omega})$ is a nonnegative function fulfilling $\int_{\Omega} x^{\beta} \ln u_{0}(x) d x$ $>-\infty$, and let $\left(\varepsilon_{j}\right)_{j \in \mathbb{N}} \subset\left(0, \varepsilon_{0}\right)$ be such that $\varepsilon_{j} \searrow 0$ as $j \rightarrow \infty$. Assume that for each $\varepsilon \in\left(\varepsilon_{j}\right)_{j \in \mathbb{N}}, u_{\varepsilon} \in$ $C^{4,1}(\bar{\Omega} \times[0, T))$ is a positive classical solution of 3.7$)$ in $\Omega \times(0, T)$ satisfying $\int_{\Omega}(x+\varepsilon)^{\beta} \ln u_{\varepsilon}(x, 0) d x \rightarrow$ $\int_{\Omega} x^{\beta} \ln u_{0}(x) d x$ and $u_{\varepsilon} \rightarrow u$ in $C_{l o c}^{0}(\bar{\Omega} \times[0, T))$ as $\varepsilon=\varepsilon_{j} \searrow 0$ for some continuous weak solution $u$ of (1.1) in $\Omega \times(0, T)$. Then $u$ is an entropy solution of (1.1) in $\Omega \times(0, T)$.

Proof. In order to verify the entropy inequality (3.6), we multiply (3.7) by $(x+\varepsilon)^{\beta} u_{\varepsilon}$ and integrate by parts twice. Since with $J \equiv J\left[u_{\varepsilon}\right]$ as in (3.9) we know from (3.10) that $J_{x}=u_{\varepsilon x}=0$ on $\partial \Omega$, we obtain

$$
\begin{aligned}
-\frac{d}{d t} \int_{\Omega}(x+\varepsilon)^{\beta} \ln u_{\varepsilon} d x= & -\int_{\Omega}(x+\varepsilon)^{\beta} \frac{u_{\varepsilon t}}{u_{\varepsilon}} d x=-\int_{\Omega} \frac{1}{u_{\varepsilon}} J_{x x} d x=-\int_{\Omega} \frac{u_{\varepsilon x}}{u_{\varepsilon}^{2}} J_{x} d x \\
= & \int_{\Omega}\left\{\frac{u_{\varepsilon x x}}{u_{\varepsilon}^{2}}-2 \frac{u_{\varepsilon x}^{2}}{u_{\varepsilon}^{3}}\right\}\left\{-g_{\varepsilon}(x) u_{\varepsilon}^{n} u_{\varepsilon x x}^{2}+2 g_{\varepsilon}(x) u_{\varepsilon}^{n-1} u_{\varepsilon x}^{2}\right\} d x \\
= & -\int_{\Omega} g_{\varepsilon}(x) u_{\varepsilon}^{n-2} u_{\varepsilon x x}^{2} d x-4 \int_{\Omega} g_{\varepsilon}(x) u_{\varepsilon}^{n-4} u_{\varepsilon x}^{4} d x \\
& +4 \int_{\Omega} g_{\varepsilon}(x) u_{\varepsilon}^{n-3} u_{\varepsilon x}^{2} u_{\varepsilon x x} d x \quad \text { for all } t \in(0, T) .
\end{aligned}
$$

Another integration by parts in the last term, using $u_{\varepsilon, x}^{2} u_{\varepsilon, x x}=\frac{1}{3}\left(u_{\varepsilon, x}^{3}\right)_{x}$, yields

$$
4 \int_{\Omega} g_{\varepsilon}(x) u_{\varepsilon}^{n-3} u_{\varepsilon x}^{2} u_{\varepsilon x x} d x=\frac{4(3-n)}{3} \int_{\Omega} g_{\varepsilon}(x) u_{\varepsilon}^{n-4} u_{\varepsilon x}^{4} d x-\frac{4}{3} \int_{\Omega} g_{\varepsilon}(x) u_{\varepsilon}^{n-3} u_{\varepsilon x}^{3} d x,
$$

so that from (3.16), we infer that

$$
\begin{aligned}
\int_{\Omega}(x+\varepsilon)^{\beta} \ln _{+} \frac{1}{u_{\varepsilon}(x, t)} d x+ & \int_{0}^{t} \int_{\Omega} g_{\varepsilon}(x) u_{\varepsilon}^{n-2} u_{\varepsilon x x}^{2} d x d s+\frac{4 n}{3} \int_{0}^{t} \int_{\Omega} g_{\varepsilon}(x) u_{\varepsilon}^{n-4} u_{\varepsilon x}^{4} d x d s \\
= & \int_{\Omega}(x+\varepsilon)^{\beta} \ln _{-} \frac{1}{u_{\varepsilon}(x, t)} d x-\int_{\Omega}(x+\varepsilon)^{\beta} \ln u_{0 \varepsilon}(x) d x \\
& -\frac{4}{3} \int_{0}^{t} \int_{\Omega} g_{\varepsilon x}(x) u_{\varepsilon}^{n-3} u_{\varepsilon x}^{3} d x d s \quad \text { for all } t \in(0, T),
\end{aligned}
$$

where $\ln _{-} z:=\max \{0,-\ln z\}$ for $z>0$. Here, according to our assumptions on the convergence of $u_{0 \varepsilon}$ and $u_{\varepsilon}$, we have

$$
\int_{\Omega}(x+\varepsilon)^{\beta} \ln _{-} \frac{1}{u_{\varepsilon}(x, t)} d x \rightarrow \int_{\Omega} x^{\beta} \ln _{-} \frac{1}{u(x, t)} d x \quad \text { for all } t \in(0, T)
$$

and

$$
-\int_{\Omega}(x+\varepsilon)^{\beta} \ln u_{0 \varepsilon}(x) d x \rightarrow-\int_{\Omega} x^{\beta} \ln u_{0}(x) d x
$$

as $\varepsilon=\varepsilon_{j} \searrow 0$, because $\beta>-1$. As for the last term in (3.17), we first use Young's inequality and Lemma 3.2 to find $c_{1}>0$ and $c_{2}>0$ such that

$$
\begin{aligned}
-\frac{4}{3} \int_{0}^{t} \int_{\Omega} g_{\varepsilon x}(x) u_{\varepsilon}^{n-3} u_{\varepsilon x}^{3} d x d s & \leq \frac{2 n}{3} \int_{0}^{t} \int_{\Omega} g_{\varepsilon}(x) u_{\varepsilon}^{n-4} u_{\varepsilon x}^{4} d x d s+c_{1} \int_{0}^{t} \int_{\Omega} \frac{g_{\varepsilon x}^{4}(x)}{g_{\varepsilon}^{3}(x)} \cdot u_{\varepsilon}^{n} d x d s \quad(3.20) \\
& \leq \frac{2 n}{3} \int_{0}^{t} \int_{\Omega} g_{\varepsilon}(x) u_{\varepsilon}^{n-4} u_{\varepsilon x}^{4} d x d s+c_{1} c_{2} \int_{0}^{t} \int_{\Omega}(x+\varepsilon)^{\alpha-4} u_{\varepsilon}^{n} d x d s .
\end{aligned}
$$


Since the uniform convergence $u_{\varepsilon}^{n} \rightarrow u^{n}$ in $\Omega \times(0, t)$ and the hypothesis $\alpha>3$ warrant that

$$
\int_{0}^{t} \int_{\Omega}(x+\varepsilon)^{\alpha-4} u_{\varepsilon}^{n} d x d s \rightarrow \int_{0}^{t} \int_{\Omega} x^{\alpha-4} u^{n} d x d s \quad \text { as } \varepsilon=\varepsilon_{j} \searrow 0,
$$

(3.17)-(3.20) imply that there exists $c_{3}(t)>0$ fulfilling

$$
\int_{0}^{t} \int_{\Omega} g_{\varepsilon}(x) u_{\varepsilon}^{n-4} u_{\varepsilon x}^{4} d x d s \leq c_{3}(t)
$$

for all $\varepsilon \in\left(\varepsilon_{j}\right)_{j \in \mathbb{N}}$. We split the last term in (3.17) in two integrals over $\{u>0\}$ and $\{u=0\}$, respectively. For the latter one, we obtain, using the Hölder inequality and (3.21), that

$$
\begin{aligned}
\left|\int_{0}^{t} \int_{\Omega} \chi_{\{u=0\}} g_{\varepsilon x}(x) u_{\varepsilon}^{n-3} u_{\varepsilon x}^{3} d x d s\right| \leq & \left(\int_{0}^{t} \int_{\Omega} g_{\varepsilon}(x) u_{\varepsilon}^{n-4} u_{\varepsilon x}^{4} d x d s\right)^{\frac{3}{4}} \\
& \times\left(\int_{0}^{t} \int_{\Omega} \chi_{\{u=0\}} \frac{g_{\varepsilon x}^{4}(x)}{g_{\varepsilon}^{3}(x)} \cdot u_{\varepsilon}^{n} d x d s\right)^{\frac{1}{4}} \\
\leq & c_{3}^{\frac{3}{4}}(t)\left(\int_{0}^{t} \int_{\Omega} \chi_{\{u=0\}}(x+\varepsilon)^{\alpha-4} u_{\varepsilon}^{n} d x d s\right)^{\frac{1}{4}} \rightarrow 0
\end{aligned}
$$

as $\varepsilon=\varepsilon_{j} \searrow 0$, because $u_{\varepsilon}^{n} \rightarrow 0$ uniformly in $(\Omega \times(0, t)) \cap\{u=0\}$ and $\frac{g_{\varepsilon x}^{4}}{g_{\varepsilon}^{3}} \rightarrow x^{\alpha-4}$ in $L^{1}(\Omega)$ according to Lemma 3.2 and the dominated convergence theorem. In order to show that moreover

$$
\int_{0}^{t} \int_{\Omega} \chi_{\{u>0\}} g_{\varepsilon x}(x) u_{\varepsilon}^{n-3} u_{\varepsilon x}^{3} d x d s \rightarrow \alpha \int_{0}^{t} \int_{\Omega} \chi_{\{u>0\}} x^{\alpha-1} u^{n-3} u_{x}^{3} d x d s
$$

as $\varepsilon=\varepsilon_{j} \searrow 0$, we first observe that thanks to (3.21), for a subsequence $\left(\varepsilon_{j_{l}}\right)_{l \in \mathbb{N}}$ and some $v \in$ $L^{\frac{4}{3}}(\Omega \times(0, t))$, we have

$$
\chi_{\{u>0\}} g_{\varepsilon}^{\frac{3}{4}} u_{\varepsilon}^{\frac{3(n-4)}{4}} u_{\varepsilon x}^{3} \rightarrow v \quad \text { in } L^{\frac{4}{3}}(\Omega \times(0, t))
$$

as $\varepsilon=\varepsilon_{j} \searrow 0$. On the other hand, the assumed uniformity of the convergence $u_{\varepsilon} \rightarrow u$ in $\Omega \times(0, t)$ together with interior parabolic regularity theory and the Arzelà-Ascoli theorem guarantee that also

$$
u_{\varepsilon} \rightarrow u \quad \text { in } C_{l o c}^{2,1}((\Omega \times(0, t)) \cap\{u>0\}) .
$$

This allows for the identification $v=\chi_{\{u>0\}} x^{\frac{3 \alpha}{4}} u^{\frac{3(n-4)}{4}} u_{x}^{3}$ in (3.24) and hence for the conclusion that (3.24) actually holds along the whole sequence $\varepsilon=\varepsilon_{j} \searrow 0$. Since furthermore Lemma 3.2 and the assumption $\alpha>3$ readily imply that

$$
g_{\varepsilon}^{-\frac{3}{4}}(x) g_{\varepsilon x}(x) \cdot u_{\varepsilon}^{\frac{n}{4}} \rightarrow x^{\frac{\alpha-4}{4}} u^{\frac{n}{4}} \quad \text { in } L^{4}(\Omega \times(0, t))
$$

as $\varepsilon=\varepsilon_{j} \searrow 0$, (3.23) results from (3.24) and (3.26) upon an obvious multiplicative decomposition in the integral on the left-hand side of (3.23).

We now insert (3.18), (3.19), (3.22), and (3.23) into the identity (3.17), and apply (3.25) and Fatou's 
lemma to the nonnegative integrals on the left-hand side of the latter to infer upon taking $\varepsilon=\varepsilon_{j} \searrow 0$ in (3.17) that

$$
\begin{aligned}
\int_{\Omega} x^{\beta} \ln _{+} \frac{1}{u(x, t)} d x+ & \int_{0}^{t} \int_{\Omega} \chi_{\{u>0\}} x^{\alpha} u^{n-2} u_{x x}^{2} d x d s+\frac{4 n}{3} \int_{0}^{t} \int_{\Omega} \chi_{\{u>0\}} x^{\alpha} u^{n-4} u_{x}^{4} d x d s \\
\leq & \int_{\Omega} x^{\beta} \ln _{-} \frac{1}{u(x, t)} d x-\int_{\Omega} x^{\beta} \ln u_{0}(x) d x \\
& -\frac{4 \alpha}{3} \int_{0}^{t} \int_{\Omega} \chi_{\{u>0\}} x^{\alpha-1} u^{n-3} u_{x}^{3} d x d s \quad \text { for all } t \in(0, T) .
\end{aligned}
$$

This in particular ensures that the regularity requirements (3.5) in Definition 3.1 are fulfilled, so that according to the boundary condition (3.2) satisfied by the continuous weak solution $u$, we may apply Lemma 3.3 to rewrite the last term in (3.27). After a straightforward rearrangement, we thereby finally conclude that $u$ indeed satisfies the entropy inequality (3.6).

\subsection{Local existence of entropy solutions}

In light of Lemma 3.4, our goal will be to construct entropy solutions of (1.1) as limits of appropriate solutions to (3.7). To prepare the framework for this in a way refining the setting in [13, let us suppose that $\beta>-1$ and $\gamma<1$ and that $u_{0} \in W_{\gamma}^{1,2}(\Omega)$ is a nonnegative function satisfying $\int_{\Omega} x^{\beta} \ln u_{0}(x) d x>$ $-\infty$. Then Lemma A.1 in the appendix ensures that whenever $\left(\varepsilon_{j}\right)_{j \in \mathbb{N}} \subset(0, \infty)$ is such that $\varepsilon_{j} \searrow 0$ as $j \rightarrow \infty$, we can construct a sequence $\left(u_{0 \varepsilon_{j}}\right)_{j \in \mathbb{N}}$ of functions $u_{0 \varepsilon_{j}} \in C^{\infty}(\bar{\Omega})$ fulfilling

$$
u_{0 \varepsilon}>0 \quad \text { in } \bar{\Omega} \quad \text { and } \quad u_{0 \varepsilon x} \in C_{0}^{\infty}(\Omega) \quad \text { for all } \varepsilon \in\left(\varepsilon_{j}\right)_{j \in \mathbb{N}}
$$

and

$$
u_{0 \varepsilon} \rightarrow u_{0} \quad \text { in } C^{0}(\bar{\Omega}) \quad \text { as } \varepsilon=\varepsilon_{j} \searrow 0
$$

as well as

$$
\int_{\Omega}(x+\varepsilon)^{\gamma} u_{0 \varepsilon x}^{2} \rightarrow \int_{\Omega} x^{\gamma} u_{0 x}^{2} \quad \text { as } \varepsilon=\varepsilon_{j} \searrow 0
$$

and

$$
\int_{\Omega}(x+\varepsilon)^{\beta} \ln u_{0 \varepsilon} \rightarrow \int_{\Omega} x^{\beta} \ln u_{0} \quad \text { as } \varepsilon=\varepsilon_{j} \searrow 0 .
$$

It has been shown in [13, Lemma 6.1] that when $n \in\left(n_{\star}, 3\right), \alpha>3, \beta \in(-1, \alpha-4)$, and $\gamma \in$ $(5-\alpha+\beta, 1)$, the properties (3.28), (3.29), and (3.30) guarantee the existence of $T>0$ such that for all suitably small $\varepsilon \in\left(\varepsilon_{j}\right)_{j \in \mathbb{N}}$, the problem (3.7) with the initial condition $u_{\varepsilon}(\cdot, 0)=u_{0 \varepsilon}$ possesses a positive classical solution $u_{\varepsilon}$ in $\Omega \times(0, T)$. Along an appropriate subsequence, these solutions approach a continuous weak solution of (1.1) in $\Omega \times(0, T)$. Under the additional hypotheses that $\beta \leq \frac{\alpha-n-3}{n}$ and that (3.31) holds, this can be sharpened as follows.

Lemma 3.5 (Local existence of an entropy solution) Let $n \in\left(n_{\star}, 3\right)$ with $n_{\star}$ as in Theorem 1.1, and let $\alpha>3, \beta \in\left(-1, \frac{\alpha-n-3}{n}\right]$, and $\gamma \in(5-\alpha+\beta, 1)$. Then one can find $K>0$ and, given $A>0$ and $B>0$, pick $T=T(A, B) \in(0,1)$ such that for any nonnegative $u_{0} \in W_{\gamma}^{1,2}(\Omega)$ satisfying

$$
\int_{\Omega} x^{\gamma} u_{0 x}^{2}(x) d x \leq A, \quad \int_{\Omega} x^{\beta} u_{0}(x) d x \leq B \quad \text { as well as } \quad \int_{\Omega} x^{\beta} \ln u_{0}(x) d x>-\infty,
$$


the problem (1.1) possesses an entropy solution $u$ in $\Omega \times(0, T)$. This solution can be obtained as the limit of positive classical solutions $u_{\varepsilon}$ of (3.7) in $\Omega \times(0, T)$ along a sequence of numbers $\left(0, \varepsilon_{0}\right) \ni \varepsilon=$ $\varepsilon_{j} \searrow 0$ as $j \rightarrow \infty$, with $u_{0 \varepsilon}:=u_{\varepsilon}(\cdot, 0)$ fulfilling (3.28)-(3.31), in the sense that

$$
u_{\varepsilon} \rightarrow u \quad \text { in } C^{0}(\bar{\Omega} \times[0, T)) \quad \text { as } \varepsilon=\varepsilon_{j} \searrow 0 .
$$

This function belongs to $L^{\infty}\left((0, T) ; W_{\gamma}^{1,2}(\Omega)\right)$ with

$$
\int_{\Omega} x^{\gamma} u_{x}^{2}(x, t) d x \leq \int_{\Omega} x^{\gamma} u_{0 x}^{2}(x) d x+K \int_{0}^{t} \int_{\Omega} x^{\alpha-\beta+\gamma-6} u^{n+2} d x d s \quad \text { for a.e. } t \in(0, T),
$$

and furthermore, we have

$$
\int_{\Omega} x^{\beta} u(x, t) d x=\int_{\Omega} x^{\beta} u_{0}(x) d x \quad \text { for all } t \in(0, T) .
$$

Proof. We first observe that since $n>1$ and $\alpha>3$, the assumption $\beta \leq \frac{\alpha-n-3}{n}$ ensures that also $\beta<\alpha-4$. As a consequence thereof, we may apply [13, Lemma 6.1] to find $T(A, B) \in(0,1)$ such that whenever the first two inequalities in (3.32) hold and $\left(\varepsilon_{j}\right)_{j \in \mathbb{N}} \subset\left(0, \varepsilon_{0}\right)$ and $\left(u_{0 \varepsilon_{j}}\right)_{j \in \mathbb{N}} \subset C^{\infty}(\bar{\Omega})$ are such that $\varepsilon_{j} \searrow 0$ as $j \rightarrow \infty$ and (3.28)-(3.30) are valid, for all sufficiently small $\varepsilon \in\left(\varepsilon_{j}\right)_{j \in \mathbb{N}}$, the problem (3.7) possesses a unique positive classical solution $u_{\varepsilon}$ in $\Omega \times(0, T(A, B))$ with $u_{\varepsilon}(\cdot, 0)=u_{0 \varepsilon}$, and for a suitable subsequence $\left(\varepsilon_{j_{k}}\right)_{k \in \mathbb{N}}$ we have $u_{\varepsilon} \rightarrow u$ in $C^{0}\left(\bar{\Omega} \times[0, T(A, B)]\right.$ as $\varepsilon=\varepsilon_{j_{k}} \searrow 0$, where $u$ is a continuous weak solution of (1.1) in $\Omega \times(0, T(A, B))$. Furthermore, this solution satisfies (3.35) due to [13, Lemma 2.3], and the inclusion $u \in L^{\infty}\left((0, T) ; W_{\gamma}^{1,2}(\Omega)\right)$ as well as (3.34) are consequences of [13, Lemma 3.1].

Now ,given $u_{0}$ satisfying (3.32), we only need to choose any sequence $\left(\varepsilon_{j}\right)_{j \in \mathbb{N}} \subset\left(0, \varepsilon_{0}\right)$ such that $\varepsilon_{j} \searrow 0$ as $j \rightarrow \infty$, use Lemma A.1 to construct $\left(u_{0 \varepsilon_{j}}\right)_{j \in \mathbb{N}} \subset C^{\infty}(\bar{\Omega})$ fulfilling (3.28)-(3.30) and (3.31), and then apply the above to find, passing to subsequences if necessary, that (3.33) holds for some continuous weak solution $u \in L^{\infty}\left((0, T) ; W_{\gamma}^{1,2}(\Omega)\right)$ satisfying (3.34) and (3.35). According to (3.31), however, we may apply Lemma 3.4 to conclude that $u$ actually is an entropy solution of (1.1) in $\Omega \times(0, T(A, B))$.

We can now prove our main result on local existence and extensibility of entropy solutions to (1.1).

Proof of Theorem 1.1. We let $K$ be as provided by Lemma 3.5 and introduce the set

$$
\begin{array}{r|l}
S:=\left\{T>0 \mid \begin{array}{l}
\text { There exists an entropy solution } u \in L^{\infty}\left((0, T) ; W_{\gamma}^{1,2}(\Omega)\right) \text { of (1.1) in } \Omega \times(0, T) \\
\text { which satisfies (3.34) and (3.35) }\} .
\end{array}\right.
\end{array}
$$

Then according to Lemma 3.5, $S$ is not empty and hence $T_{\max }:=\sup S \in(0, \infty]$ well-defined. Now if $T_{\max }<\infty$ but $u \leq M$ in $\Omega \times\left(0, T_{\max }\right)$ for some $M>0$, then by (3.34) and the fact that $\alpha-\beta+\gamma-6>-1$, we could find a null set $N \subset\left(0, T_{\max }\right)$ such that

$$
\int_{\Omega} x^{\gamma} u_{x}^{2}(x, t) d x \leq A_{0}:=\int_{\Omega} x^{\gamma} u_{0 x}^{2}(x) d x+K M^{n+2} T_{\max } \int_{\Omega} x^{\alpha-\beta+\gamma-6} d x \quad \text { for all } t \in\left(0, T_{\max }\right) \backslash N,
$$

Upon another application of Lemma 3.5 to $A:=A_{0}$ and $B:=\int_{\Omega} x^{\beta} u_{0}(x) d x$, and with $u_{0}$ replaced by $u\left(\cdot, t_{0}\right)$ for fixed $t_{0} \in\left(0, T_{\max }\right) \backslash N$ such that $t_{0}>T_{\max }-\frac{1}{2} T\left(A_{0}, B_{0}\right)$, we would thus readily obtain that $u$ can be extended as an entropy solution of (1.1) in $\Omega \times\left(0, t_{0}+T\left(A_{0}, B_{0}\right)\right)$, yet belonging to $L^{\infty}\left(\left(0, t_{0}+T\left(A_{0}, B_{0}\right)\right) ; W_{\gamma}^{1,2}(\Omega)\right)$ and satisfying (3.34) and (3.35). This contradiction to the definition of $T_{\max }$ implies that actually (1.4) must be valid. 


\section{Blow-up}

\subsection{Additional reqularity properties of entropy solutions}

As a first step toward our blow-up proof, we show the following consequence of the entropy inequality (3.6).

Lemma 4.1 (Consequence of entropy inequality) Let $n>1, \alpha>3$, and $\beta \in\left(-1, \frac{\alpha-n-3}{n}\right.$ ]. Then there exists $C>0$ such that if $u_{0} \in C^{0}(\bar{\Omega})$ is nonnegative and satisfies $\int_{\Omega} x^{\beta} \ln u_{0}(x) d x>-\infty$, and if $u$ is an entropy solution of (1.1) in $\Omega \times(0, T)$ for some $T>0$, the inequality

$$
\begin{aligned}
\int_{0}^{t} \int_{\Omega} \chi_{\{u>0\}} x^{\alpha} u^{n-2} u_{x x}^{2} d x d s & +\int_{0}^{t} \int_{\Omega} \chi_{\{u>0\}} x^{\alpha} u^{n-4} u_{x}^{4} d x d s \\
\leq C & \left\{\int_{\Omega} x^{\beta} \ln _{+} \frac{1}{u_{0}(x)} d x+\int_{\Omega} x^{\beta} u_{0}(x) d x+t \cdot\left(\int_{\Omega} x^{\beta} u_{0}(x) d x\right)^{n}\right\}
\end{aligned}
$$

is valid for all $t \in(0, T)$.

Proof. In view of Lemma 3.3. we can rewrite the entropy inequality (3.6) in the form

$$
\begin{aligned}
-\int_{\Omega} x^{\beta} \ln u(x, t) d x & +\int_{0}^{t} \int_{\Omega} \chi_{\{u>0\}} x^{\alpha} u^{n-2} u_{x x}^{2} d x d s+\frac{4 n}{3} \int_{0}^{t} \int_{\Omega} \chi_{\{u>0\}} x^{\alpha} u^{n-4} u_{x}^{4} d x d s \\
& \leq-\int_{\Omega} x^{\beta} \ln u_{0}(x) d x-\frac{4 \alpha}{3} \int_{0}^{t} \int_{\Omega} \chi_{\{u>0\}} x^{\alpha-1} u^{n-3} u_{x}^{3} d x d s,
\end{aligned}
$$

where the last term can be estimated using Young's inequality so as to obtain

$$
-\frac{4 \alpha}{3} \int_{0}^{t} \int_{\Omega} \chi_{\{u>0\}} x^{\alpha-1} u^{n-3} u_{x}^{3} d x d s \leq \frac{2 n}{3} \int_{0}^{t} \int_{\Omega} \chi_{\{u>0\}} x^{\alpha} u^{n-4} u_{x}^{4} d x d s+\frac{3 \alpha^{4}}{2 n^{3}} \int_{0}^{t} \int_{\Omega} x^{\alpha-4} u^{n} d x d s
$$

for $t \in(0, T)$. Now thanks to the assumption $\beta \leq \frac{\alpha-n-3}{n}$, we may invoke Lemma 2.3 to find $c_{1}>0$ such that

$$
\frac{3 \alpha^{4}}{2 n^{3}} \int_{0}^{t} \int_{\Omega} x^{\alpha-4} u^{n} d x d s \leq \frac{n}{3} \int_{0}^{t} \int_{\Omega} \chi_{\{u>0\}} x^{\alpha} u^{n-4} u_{x}^{4} d x d s+c_{1} \int_{0}^{t}\left(\int_{\Omega} x^{\beta} u d x\right)^{n} d s
$$

for all such $t$. Recalling (3.4) and using the fact that $\ln \xi \leq \xi$ for all $\xi>0$, we thereby infer that

$$
\begin{aligned}
\int_{0}^{t} \int_{\Omega} \chi_{\{u>0\}} x^{\alpha} u^{n-2} u_{x x}^{2} d x d s & +\frac{n}{3} \int_{0}^{t} \int_{\Omega} \chi_{\{u>0\}} x^{\alpha} u^{n-4} u_{x}^{4} d x d s \\
& \leq-\int_{\Omega} x^{\beta} \ln u_{0}(x) d x+\int_{\Omega} x^{\beta} \ln u(x, t) d x+c_{1}\left(\int_{\Omega} x^{\beta} u_{0}(x) d x\right)^{n} \cdot t \\
& \leq \int_{\Omega} x^{\beta} \ln _{+} \frac{1}{u_{0}(x)} d x+\int_{\Omega} x^{\beta} u_{0}(x) d x+c_{1}\left(\int_{\Omega} x^{\beta} u_{0}(x) d x\right)^{n} \cdot t
\end{aligned}
$$

which proves (4.1).

The following additional regularity properties of any entropy solution are consequences of the above lemma. 
Corollary 4.2 (Regularity of entropy solutions) Let $n>1, \alpha>3$, and $\beta \in\left(-1, \frac{\alpha-n-3}{n}\right]$. Suppose that $u_{0} \in C^{0}(\bar{\Omega})$ is nonnegative with $\int_{\Omega} x^{\beta} \ln u_{0}(x) d x>-\infty$, and that $u$ is an entropy solution of (1.1) in $\Omega \times(0, T)$ for some $T>0$. Then for any $\lambda \in\left(-\infty, \frac{\alpha+1}{2}\right)$ and each fixed $t \in(0, T)$, we have

$$
\int_{0}^{t} \int_{\Omega} \chi_{\{u>0\}} x^{\alpha-\lambda} u^{n}\left|u_{x x}\right| d x d s<\infty
$$

and

$$
\int_{0}^{t} \int_{\Omega} \chi_{\{u>0\}} x^{\alpha-\lambda} u^{n-1} u_{x}^{2} d x d s<\infty
$$

as well as

$$
\int_{0}^{t} \int_{\Omega} \chi_{\{u>0\}} x^{\alpha-\frac{3}{2} \lambda} u^{n}\left|u_{x}\right| d x d s<\infty .
$$

Proof. By Young's inequality, we have

$$
\int_{0}^{t} \int_{\Omega} \chi_{\{u>0\}} x^{\alpha-\lambda} u^{n}\left|u_{x x}\right| d x d s \leq \int_{0}^{t} \int_{\Omega} \chi_{\{u>0\}} x^{\alpha} u^{n-2} u_{x x}^{2} d x d s+\int_{0}^{t} \int_{\Omega} x^{\alpha-2 \lambda} u^{n+2} d x d s
$$

and

$$
\int_{0}^{t} \int_{\Omega} \chi_{\{u>0\}} x^{\alpha-\lambda} u^{n-1} u_{x}^{2} d x d s \leq \int_{0}^{t} \int_{\Omega} \chi_{\{u>0\}} x^{\alpha} u^{n-4} u_{x}^{4} d x d s+\int_{0}^{t} \int_{\Omega} x^{\alpha-2 \lambda} u^{n+2} d x d s
$$

as well as

$$
\int_{0}^{t} \int_{\Omega} \chi_{\{u>0\}} x^{\alpha-\frac{3}{2} \lambda} u^{n}\left|u_{x}\right| d x d s \leq \int_{0}^{t} \int_{\Omega} \chi_{\{u>0\}} x^{\alpha} u^{n-4} u_{x}^{4} d x d s+\int_{0}^{t} \int_{\Omega} x^{\alpha-2 \lambda} u^{n+\frac{4}{3}} d x d s .
$$

Here, thanks to the continuity of $u$ in $\bar{\Omega} \times[0, t]$ and the fact that $\alpha-2 \lambda>-1$ by assumption on $\lambda$, the rightmost integrals are finite. Therefore, the assertion is an immediate consequence of Lemma 4.1 .

\subsection{Integration by parts}

For the proof of Theorem 1.2, we need two more integration-by-parts formulae, as well as a consequence thereof, which we can verify for entropy solutions.

Lemma 4.3 (Integration by parts II) Let $n>1, \alpha>3, \beta \in\left(-1, \frac{\alpha-n-3}{n}\right]$, and $\kappa<\frac{1}{2}(\alpha-3)$. Assume that $u_{0} \in C^{0}(\bar{\Omega})$ is nonnegative and satisfies $\int_{\Omega} x^{\alpha} \ln u_{0}(x) d x>-\infty$, and that $u$ is an entropy solution of (1.1) in $\Omega \times(0, T)$ for some $T>0$. Moreover, let $\psi \in C^{1}(\bar{\Omega})$ be nonnegative and such that $\psi(L)=0$.

(i) For each $t_{0} \in(0, T)$, the identity

$$
\begin{aligned}
\int_{0}^{t_{0}} \int_{\Omega} \psi(x) x^{\alpha-\kappa-4} u^{n+1} d x d s= & -\frac{n+1}{\alpha-\kappa-3} \int_{0}^{t_{0}} \int_{\Omega} \chi_{\{u>0\}} \psi(x) x^{\alpha-\kappa-3} u^{n} u_{x} d x d s \\
& -\frac{1}{\alpha-\kappa-3} \int_{0}^{t_{0}} \int_{\Omega} \psi_{x}(x) x^{\alpha-\kappa-3} u^{n+1} d x d s
\end{aligned}
$$


and the inequality

$$
\begin{aligned}
\int_{0}^{t_{0}} \int_{\Omega} \psi(x) x^{\alpha-\kappa-4} u^{n+1} d x d s \leq & \left(\frac{n+1}{\alpha-\kappa-3}\right)^{2} \int_{0}^{t_{0}} \int_{\Omega} \chi_{\{u>0\}} \psi(x) x^{\alpha-\kappa-2} u^{n-1} u_{x}^{2} d x d s \\
& -\frac{2}{\alpha-\kappa-3} \int_{0}^{t_{0}} \int_{\Omega} \psi_{x}(x) x^{\alpha-\kappa-3} u^{n+1} d x d s
\end{aligned}
$$

hold.

(ii) For all $t_{0} \in(0, T)$, we have

$$
\begin{aligned}
\int_{0}^{t_{0}} \int_{\Omega} \chi_{\{u>0\}} \psi(x) x^{\alpha-\kappa-2} u^{n} u_{x x} d x d s= & -n \int_{0}^{t_{0}} \int_{\Omega} \chi_{\{u>0\}} \psi(x) x^{\alpha-\kappa-2} u^{n-1} u_{x}^{2} d x d s \\
& +\frac{(\alpha-\kappa-2)(\alpha-\kappa-3)}{n+1} \int_{0}^{t_{0}} \int_{\Omega} \psi(x) x^{\alpha-\kappa-4} u^{n+1} d x d s \\
& -\int_{0}^{t_{0}} \int_{\Omega} \chi_{\{u>0\}} \psi_{x}(x) x^{\alpha-\kappa-2} u^{n} u_{x} d x d s \\
& +\frac{\alpha-\kappa-2}{n+1} \int_{0}^{t_{0}} \int_{\Omega} \psi_{x}(x) x^{\alpha-\kappa-3} u^{n+1} d x d s .
\end{aligned}
$$

Proof. As in the proof of Lemma 3.3 , we fix some $\zeta \in C^{\infty}(\mathbb{R})$ fulfilling $\zeta \equiv 0$ on $(-\infty, 1]$ and $\zeta \equiv 1$ on $[2, \infty)$ as well as $0 \leq \zeta^{\prime} \leq 2$, and let

$$
\chi_{\delta}(s):=\zeta\left(\frac{s}{\delta}\right), \quad s \geq 0
$$

for $\delta>0$.

(i) In order to prove (4.2), we note that since $\kappa<\frac{1}{2}(\alpha-3)<\alpha-3$ and $u$ is smooth in $([\eta, L] \times$ $\left.\left[\tau, t_{0}\right]\right) \cap\{u>0\}$ for all $\eta \in(0, L)$ and $\tau \in\left(0, t_{0}\right)$, in each of the expressions

$$
I(\eta, \tau, \delta):=\int_{\tau}^{t_{0}} \int_{\Omega} \psi(x)(x-\eta)_{+}^{\alpha-\kappa-4} \chi_{\delta}(u) u^{n+1} d x d s
$$

we may integrate by parts to find that

$$
\begin{aligned}
I(\eta, \tau, \delta)= & \frac{1}{\alpha-\kappa-3} \int_{\tau}^{t_{0}} \int_{\Omega} \psi(x)\left((x-\eta)_{+}^{\alpha-\kappa-3}\right)_{x} \chi_{\delta}(u) u^{n+1} d x d s \\
= & -\frac{n+1}{\alpha-\kappa-3} \int_{\tau}^{t_{0}} \int_{\Omega} \psi(x)(x-\eta)_{+}^{\alpha-\kappa-3} \chi_{\delta}(u) u^{n} u_{x} d x d s \\
& -\frac{1}{\alpha-\kappa-3} \int_{\tau}^{t_{0}} \int_{\Omega} \psi(x)(x-\eta)_{+}^{\alpha-\kappa-3} \chi_{\delta}^{\prime}(u) u^{n+1} u_{x} d x d s \\
& -\frac{1}{\alpha-\kappa-3} \int_{\tau}^{t_{0}} \int_{\Omega} \psi_{x}(x)(x-\eta)_{+}^{\alpha-\kappa-3} \chi_{\delta}(u) u^{n+1} d x d s \\
=: & I_{1}(\eta, \tau, \delta)+I_{2}(\eta, \tau, \delta)+I_{3}(\eta, \tau, \delta) .
\end{aligned}
$$


In order to prepare the limit process $\delta \searrow 0$, we note that

$$
\chi_{\delta}^{\prime}(u) \equiv 0 \quad \text { in }(\Omega \times(0, T)) \backslash\{\delta<u<2 \delta\},
$$

that

$$
0 \leq \chi_{\delta}^{\prime} \leq \frac{2}{\delta} \quad \text { in }[0, \infty)
$$

and that hence

$$
\left|u \chi_{\delta}^{\prime}(u)\right| \leq 4 \quad \text { in } \Omega \times\left(0, t_{0}\right) .
$$

Next, since $\psi$ and $\psi_{x}$ are continuous in $\bar{\Omega}$ and $u$ is continuous in $\bar{\Omega} \times\left[0, t_{0}\right]$, the fact that $\kappa<\alpha-2$ guarantees that

$$
\psi(x)(x-\eta)_{+}^{\alpha-\kappa-4} u^{n+1} \in L^{1}\left(\Omega \times\left(0, t_{0}\right)\right) \quad \text { for all } \eta \in(0, L)
$$

and also

$$
\left|\psi_{x}(x)\right| x^{\alpha-\kappa-3} u^{n+1} \in L^{1}\left(\Omega \times\left(0, t_{0}\right)\right) .
$$

As $\kappa<\frac{1}{2}(\alpha-3)$ and hence $\frac{2}{3}(\kappa+3)<\frac{\alpha+1}{2}$, Corollary 4.2 with $\lambda=\frac{2}{3}(\kappa+3)$ says that moreover

$$
\Omega \times\left(0, t_{0}\right) \ni(x, t) \mapsto \psi(x) x^{\alpha-\kappa-3} u^{n}\left|u_{x}\right| \in L^{1}\left(\Omega \times\left(0, t_{0}\right)\right) .
$$

Since $(x-\eta)_{+}^{\alpha-\kappa-3} \leq x^{\alpha-\kappa-3}$ and $\chi_{\delta}(u) \nearrow \chi_{\{u>0\}}$ a.e. in $\Omega \times\left(0, t_{0}\right)$ as $\delta \searrow 0$, an application of the dominated convergence theorem along with (4.9), (4.10), (4.11), and (4.12) thus ensures that for fixed $\eta \in(0, L)$ and $\tau \in\left(0, t_{0}\right)$, we may take $\delta \searrow 0$ in $I(\eta, \tau, \delta)$ and $I_{i}(\eta, \tau, \delta), i \in\{1,2,3\}$, to infer from (4.6) that

$$
\begin{aligned}
\int_{\tau}^{t_{0}} \int_{\Omega} \psi(x)(x-\eta)_{+}^{\alpha-\kappa-4} u^{n+1} d x d s= & -\frac{n+1}{\alpha-\kappa-3} \int_{\tau}^{t_{0}} \int_{\Omega} \chi_{\{u>0\}} \psi(x)(x-\eta)_{+}^{\alpha-\kappa-3} u^{n} u_{x} d x d s \\
& -\frac{1}{\alpha-\kappa-3} \int_{\tau}^{t_{0}} \int_{\Omega} \psi_{x}(x)(x-\eta)_{+}^{\alpha-\kappa-3} u^{n+1} d x d s .
\end{aligned}
$$

Here again by (4.11), (4.12), and Lebesgue's theorem, we may next let $\eta \searrow 0$ in both expressions on the right-hand side, whereas on the left-hand side we apply the Beppo-Levi theorem so as to infer from (4.13) that

$$
\begin{aligned}
\int_{\tau}^{t_{0}} \int_{\Omega} \psi(x) x^{\alpha-\kappa-4} u^{n+1} d x d s= & -\frac{n+1}{\alpha-\kappa-3} \int_{\tau}^{t_{0}} \int_{\Omega} \chi_{\{u>0\}} \psi(x) x^{\alpha-\kappa-3} u^{n} u_{x} d x d s \\
& -\frac{1}{\alpha-\kappa-3} \int_{\tau}^{t_{0}} \int_{\Omega} \psi_{x}(x) x^{\alpha-\kappa-3} u^{n+1} d x d s .
\end{aligned}
$$

Finally, quite similar arguments allow us to take $\tau \searrow 0$ in each of the integrals here and thereby conclude that (4.2) indeed holds. From this, (4.3) immediately follows upon an application of Young's inequality in estimating the first term on the right-hand side of (4.2) according to

$$
\begin{aligned}
& -\frac{n+1}{\alpha-\kappa-3} \int_{0}^{t_{0}} \int_{\Omega} \chi_{\{u>0\}} \psi(x) x^{\alpha-\kappa-3} u^{n} u_{x} d x d s \\
& \leq \frac{1}{2} \int_{0}^{t_{0}} \int_{\Omega} \psi(x) x^{\alpha-\kappa-4} u^{n+1} d x d s+\frac{1}{2}\left(\frac{n+1}{\alpha-\kappa-3}\right)^{2} \int_{0}^{t_{0}} \int_{\Omega} \chi_{\{u>0\}} \psi(x) x^{\alpha-\kappa-2} u^{n-1} u_{x}^{2} d x d s .
\end{aligned}
$$


(ii) Adapting the above approximation procedure, for $\delta>0, \eta \in(0, L)$, and $\tau \in\left(0, t_{0}\right)$, we let $\chi_{\delta}$ be as in (4.5) and then may integrate by parts to find that

$$
\begin{aligned}
\int_{\tau}^{t_{0}} \int_{\Omega} & \psi(x)(x-\eta)_{+}^{\alpha-\kappa-2} \chi_{\delta}(u) u^{n} u_{x x} d x d s=-n \int_{\tau}^{t_{0}} \int_{\Omega} \psi(x)(x-\eta)_{+}^{\alpha-\kappa-2} \chi_{\delta}(u) u^{n-1} u_{x}^{2} d x d s \\
& -\int_{\tau}^{t_{0}} \int_{\Omega} \psi(x)(x-\eta)_{+}^{\alpha-\kappa-2} \chi_{\delta}^{\prime}(u) u^{n} u_{x}^{2} d x d s \\
& -(\alpha-\kappa-2) \int_{\tau}^{t_{0}} \int_{\Omega} \psi(x)(x-\eta)_{+}^{\alpha-\kappa-3} \chi_{\delta}(u) u^{n} u_{x} d x d s \\
& -\int_{\tau}^{t_{0}} \int_{\Omega} \psi_{x}(x)(x-\eta)_{+}^{\alpha-\kappa-2} \chi_{\delta}(u) u^{n} u_{x} d x d s .
\end{aligned}
$$

For the subsequent limit procedures, we note that an application of Corollary 4.2 to $\lambda=\kappa+2<\frac{\alpha+1}{2}$ (using $\kappa<\frac{1}{2}(\alpha-3)$ ) yields the inclusions

$$
\chi_{\{u>0\}} \psi(x) x^{\alpha-\kappa-2} u^{n}\left|u_{x x}\right| \in L^{1}\left(\Omega \times\left(0, t_{0}\right)\right)
$$

and

$$
\chi_{\{u>0\}} \psi(x) x^{\alpha-\kappa-2} u^{n-1} u_{x}^{2} \in L^{1}\left(\Omega \times\left(0, t_{0}\right)\right) .
$$

Again by means of the inequality $\kappa<\frac{1}{2}(\alpha-3)$, Corollary 4.2 applied to $\lambda=\frac{2(\kappa+3)}{3}<\frac{\alpha+1}{2}$ says that moreover

$$
\chi_{\{u>0\}} \psi(x) x^{\alpha-\kappa-3} u^{n}\left|u_{x}\right| \in L^{1}\left(\Omega \times\left(0, t_{0}\right)\right),
$$

whence clearly also

$$
\chi_{\{u>0\}}\left|\psi_{x}(x)\right| x^{\alpha-\kappa-2} u^{n}\left|u_{x}\right| \in L^{1}\left(\Omega \times\left(0, t_{0}\right)\right) .
$$

Now, as for the second integral on the right-hand side of (4.14), we recall (4.7) and (4.8) to see that

$$
\varphi_{\delta}(x, t):=\psi(x)(x-\eta)_{+}^{\alpha-\kappa-2} \chi_{\delta}^{\prime}(u) u^{n} u_{x}^{2}, \quad x \in \Omega \times\left(0, t_{0}\right),
$$

satisfies $\varphi_{\delta} \rightarrow 0$ a.e. in $\Omega \times\left(0, t_{0}\right)$ as $\delta \searrow 0$ and

$$
\begin{aligned}
\left|\varphi_{\delta}(x, t)\right| & \leq\|\psi\|_{L^{\infty}(\Omega)} \cdot x^{\alpha-\kappa-2} \cdot \frac{2}{\delta} \cdot \chi_{\{\delta<u<2 \delta\}} \cdot u^{n} u_{x}^{2} \\
& \leq 4\|\psi\|_{L^{\infty}(\Omega)} \cdot x^{\alpha-\kappa-2} \chi_{\{u>0\}} u^{n-1} u_{x}^{2} \quad \text { for all } x \in \Omega, t \in\left(0, t_{0}\right), \text { and } \delta>0
\end{aligned}
$$

again because $\kappa<\frac{1}{2}(\alpha-3)<\alpha-2$. Using (4.16), from the dominated convergence theorem, we thus infer that

$$
-\int_{\tau}^{t_{0}} \int_{\Omega} \psi(x)(x-\eta)_{+}^{\alpha-\kappa-2} \chi_{\delta}^{\prime}(u) u^{n} u_{x}^{2} d x d s \rightarrow 0 \quad \text { as } \delta \searrow 0 .
$$

We next use (4.15)-(4.18) along with the fact that $\chi_{\delta}(u) \nearrow \chi_{\{u>0\}}$ as $\delta \searrow 0$ to see upon several further applications of the dominated convergence theorem that, after taking $\delta \searrow 0$, then $\eta \searrow 0$, and 
eventually $\tau \searrow 0$, (4.14) becomes

$$
\begin{aligned}
\int_{0}^{t_{0}} \int_{\Omega} \chi_{\{u>0\}} \psi(x) x^{\alpha-\kappa-2} u^{n} u_{x x} d x d s= & -n \int_{0}^{t_{0}} \int_{\Omega} \chi_{\{u>0\}} \psi(x) x^{\alpha-\kappa-2} u^{n-1} u_{x}^{2} d x d s \\
& -(\alpha-\kappa-2) \int_{0}^{t_{0}} \int_{\Omega} \chi_{\{u>0\}} \psi(x) x^{\alpha-\kappa-3} u^{n} u_{x} d x d s \\
& -\int_{0}^{t_{0}} \int_{\Omega} \chi_{\{u>0\}} \psi_{x}(x) x^{\alpha-\kappa-2} u^{n} u_{x} d x d s .
\end{aligned}
$$

Since here the second term on the right-hand side can be rewritten by means of (4.2) according to

$$
\begin{aligned}
-(\alpha-\kappa-2) & \int_{0}^{t_{0}} \int_{\Omega} \chi_{\{u>0\}} \psi(x) x^{\alpha-\kappa-3} u^{n} u_{x} d x d s \\
= & \frac{(\alpha-\kappa-2)(\alpha-\kappa-3)}{n+1} \int_{0}^{t_{0}} \int_{\Omega} \psi(x)+x^{\alpha-\kappa-4} u^{n+1} d x d s \\
& +\frac{\alpha-\kappa-2}{n+1} \int_{0}^{t_{0}} \int_{\Omega} \psi_{x}(x) x^{\alpha-\kappa-3} u^{n+1} d x d s,
\end{aligned}
$$

we infer that (4.19) implies (4.4).

\subsection{An integral inequality for $t \mapsto \int_{\Omega} x^{\beta-\kappa} u(x, t) d x$}

The core of our blow-up proof will consist of an inequality for the function $t \mapsto y(t)=\int_{\Omega} x^{\beta-\kappa} u(x, t) d x$, which we shall derive in the following lemma.

Lemma 4.4 (Integral inequality for $y(t)$ ) Let $n>1$ as well as

$$
\alpha>n+4 \quad \text { and } \quad \beta \in\left(\frac{\alpha-n-4}{n+1}, \frac{\alpha-n-3}{n}\right],
$$

and suppose that $\kappa>0$ is such that

$$
\kappa<\min \left\{\frac{\alpha-3}{2}, \alpha-n-4, \beta+1, \frac{-\alpha+(n+1) \beta+n+4}{n}\right\} .
$$

Then, given $B>0$ and $D>0$, we can find constants $C_{1}(B, D)>0, C_{2}>0$, and $C_{3}>0$ with the following property: If for some $T>0, u$ is an entropy solution of (1.1) in $\Omega \times(0, T)$ with nonnegative initial data $u_{0} \in C^{0}(\bar{\Omega})$ fulfilling

$$
\int_{\Omega} x^{\beta} u_{0}(x) d x \leq B \quad \text { and } \quad \int_{\Omega} x^{\beta} \ln _{+} \frac{1}{u_{0}(x)} d x \leq D,
$$

then

$$
y(t):=\int_{\Omega} x^{\beta-\kappa} u(x, t) d x, \quad t \in[0, T],
$$

defines a continuous function which satisfies

$$
y(t) \geq \int_{0}^{\frac{L}{4}} x^{\beta-\kappa} u_{0}(x) d x-C_{1}(B, D) \cdot(1+T)+C_{2} \int_{0}^{t}\left(y(s)-C_{3} B\right)_{+}^{n+1} d s \quad \text { for all } t \in(0, T) .
$$


Remark. As can easily be checked, (4.20) guarantees that the first and last requirements implicitly contained in (4.21) can indeed be fulfilled simultaneously for some $\kappa>0$.

Proof. $\quad$ Step 1. Let us first construct a suitable test function for Definition 3.1.

We fix a nonincreasing cut-off function $\zeta \in C^{\infty}(\mathbb{R})$ such that $\zeta \equiv 1$ in $(-\infty, 1]$ and $\zeta \equiv 0$ in $[2, \infty)$, and let

$$
\psi(x):=\zeta\left(\frac{4 x}{L}\right), \quad x \in \bar{\Omega} .
$$

As in the proof of Lemma 3.1, given $t_{0} \in(0, T)$ we moreover introduce

$$
\xi_{\delta}(t):=\zeta\left(\frac{t-t_{0}}{\delta}\right), \quad t \in[0, T]
$$

for $\delta \in\left(0, \frac{T-t_{0}}{2}\right)$. Then for any such $\delta$ and each $\eta>0$,

$$
\phi(x, t):=\xi_{\delta}(t) \psi(x)(x+\eta)^{-\kappa}, \quad x \in \bar{\Omega}, t \in[0, T],
$$

defines a function $\phi \in C_{0}^{\infty}(\bar{\Omega} \times[0, T))$ satisfying $\phi_{x}(L, t)=0$ for all $t \in(0, T)$, since $\psi \equiv 0$ in $\left.\left[L, \frac{L}{2}\right]\right)$. Accordingly, $\phi$ is an admissible test function in Definition 3.1, so that (3.3) yields

$$
\begin{aligned}
-\int_{0}^{T} \int_{\Omega} \xi_{\delta}^{\prime}(t) & \psi(x) x^{\beta}(x+\eta)^{-\kappa} u(x, t) d x d t-\int_{\Omega} \psi(x) x^{\beta}(x+\eta)^{-\kappa} u_{0}(x) d x \\
= & \int_{0}^{T} \int_{\Omega} \chi_{\{u>0\}} \xi_{\delta}(t)\left\{\psi(x)(x+\eta)^{-\kappa}\right\}_{x x}\left\{-x^{\alpha} u^{n} u_{x x}+2 x^{\alpha} u^{n-1} u_{x}^{2}\right\} d x d t
\end{aligned}
$$

for all $\delta \in\left(0, \frac{T-t_{0}}{2}\right)$.

Step 2. We next let $\delta \searrow 0$.

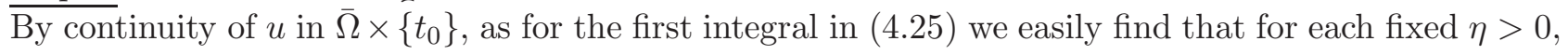

$$
\begin{aligned}
-\int_{0}^{T} \int_{\Omega} \xi_{\delta}^{\prime}(t) \psi(x) x^{\beta}(x+\eta)^{-\kappa} u(x, t) d x d t & =-\frac{1}{\delta} \int_{t_{0}+\delta}^{t_{0}+2 \delta} \int_{\Omega} \zeta^{\prime}\left(\frac{t-t_{0}}{\delta}\right) \psi(x) x^{\beta}(x+\eta)^{-\kappa} u(x, t) d x d t \\
& \rightarrow \int_{\Omega} \psi(x) x^{\beta}(x+\eta)^{-\kappa} u\left(x, t_{0}\right) d x \quad \text { as } \delta \searrow 0,
\end{aligned}
$$

whereas the integrability properties of $x^{\alpha} u^{n} u_{x x}$ and of $x^{\alpha} u^{n-1} u_{x}^{2}$ from Definition 3.1 ensure that

$$
\begin{aligned}
\int_{0}^{T} \int_{\Omega} \chi_{\{u>0\}} \xi_{\delta}(t)\left\{\psi(x)(x+\eta)^{-\kappa}\right\}_{x x}\left\{-x^{\alpha} u^{n} u_{x x}+2 x^{\alpha} u^{n-1} u_{x}^{2}\right\} d x d t & \\
& \rightarrow \int_{0}^{t_{0}} \int_{\Omega} \chi_{\{u>0\}}\left\{\psi(x)(x+\eta)^{-\kappa}\right\}_{x x}\left\{-x^{\alpha} u^{n} u_{x x}+2 x^{\alpha} u^{n-1} u_{x}^{2}\right\} d x d t \quad \text { as } \delta \searrow 0 .
\end{aligned}
$$

Computing

$$
\left\{\psi(x)(x+\eta)^{-\kappa}\right\}_{x x}=\kappa(\kappa+1) \psi(x)(x+\eta)^{-\kappa-2}-2 \kappa \psi_{x}(x)(x+\eta)^{-\kappa-1}+\psi_{x x}(x)(x+\eta)^{-\kappa}
$$


for $x \in \Omega$, we infer from (4.25) in the limit $\delta \searrow 0$ the identity

$$
\begin{aligned}
\int_{\Omega} \psi(x) x^{\beta}(x+\eta)^{-\kappa} u\left(x, t_{0}\right) d x & =\int_{\Omega} \psi(x) x^{\beta}(x+\eta)^{-\kappa} u_{0}(x) d x \\
& -\kappa(\kappa+1) \int_{0}^{t_{0}} \int_{\Omega} \chi_{\{u>0\}} \psi(x) x^{\alpha}(x+\eta)^{-\kappa-2} u^{n} u_{x x} d x d s \\
& +2 \kappa(\kappa+1) \int_{0}^{t_{0}} \int_{\Omega} \chi_{\{u>0\}} \psi(x) x^{\alpha}(x+\eta)^{-\kappa-2} u^{n-1} u_{x}^{2} d x d s \\
+ & \int_{0}^{t_{0}} \int_{\Omega} \chi_{\{u>0\}}\left\{-2 \kappa \psi_{x}(x) x^{\alpha}(x+\eta)^{-\kappa-1}+\psi_{x x}(x) x^{\alpha}(x+\eta)^{-\kappa}\right\} \\
& \times\left\{-u^{n} u_{x x}+2 u^{n-1} u_{x}^{2}\right\} d x d t .
\end{aligned}
$$

Step 3. We proceed by passing to the limit $\eta \searrow 0$.

$\overline{\text { Since } \beta}-\kappa>-1$ by (4.21) and since $\psi$ is bounded, the continuity of $u\left(\cdot, t_{0}\right)$ and of $u_{0}$ in $\bar{\Omega}$ allow us to apply the dominated convergence theorem in the first two terms in (4.26) to see that as $\eta \searrow 0$, we have

$$
\int_{\Omega} \psi(x) x^{\beta}(x+\eta)^{-\kappa} u\left(x, t_{0}\right) d x \rightarrow \int_{\Omega} \psi(x) x^{\beta-\kappa} u\left(x, t_{0}\right) d x
$$

and

$$
\int_{\Omega} \psi(x) x^{\beta}(x+\eta)^{-\kappa} u_{0}(x) d x \rightarrow \int_{\Omega} \psi(x) x^{\beta-\kappa} u_{0}(x) d x .
$$

In order to invoke a similar argument for the space-time integrals of the right-hand side of (4.26), we first observe that according to (4.22) and Corollary 4.2,

$$
\chi_{\{u>0\}} x^{\alpha-\kappa-2} u^{n}\left|u_{x x}\right| \in L^{1}\left(\Omega \times\left(0, t_{0}\right)\right)
$$

and

$$
\chi_{\{u>0\}} x^{\alpha-\kappa-2} u^{n-1} u_{x}^{2} \in L^{1}\left(\Omega \times\left(0, t_{0}\right)\right),
$$

because $\kappa+2<\frac{\alpha+1}{2}$ thanks to (4.20). Consequently, another application of the dominated convergence theorem ensures that as $\eta \searrow 0$,

$$
\begin{aligned}
-\kappa(\kappa+1) & \int_{0}^{t_{0}} \int_{\Omega} \chi_{\{u>0\}} \psi(x) x^{\alpha}(x+\eta)^{-\kappa-2} u^{n} u_{x x} d x d s \\
\rightarrow & -\kappa(\kappa+1) \int_{0}^{t_{0}} \int_{\Omega} \chi_{\{u>0\}} \psi(x) x^{\alpha-\kappa-2} u^{n} u_{x x} d x d s
\end{aligned}
$$

and

$$
\begin{aligned}
2 \kappa(\kappa+1) & \int_{0}^{t_{0}} \int_{\Omega} \chi_{\{u>0\}} \psi(x) x^{\alpha}(x+\eta)^{-\kappa-2} u^{n-1} u_{x}^{2} d x d s \\
& \rightarrow 2 \kappa(\kappa+1) \int_{0}^{t_{0}} \int_{\Omega} \chi_{\{u>0\}} \psi(x) x^{\alpha-\kappa-2} u^{n-1} u_{x}^{2} d x d s
\end{aligned}
$$


as well as

$$
\begin{gathered}
\int_{0}^{t_{0}} \int_{\Omega} \chi_{\{u>0\}}\left\{-2 \kappa \psi_{x}(x) x^{\alpha}(x+\eta)^{-\kappa-1}+\psi_{x x}(x) x^{\alpha}(x+\eta)^{-\kappa}\right\}\left\{-u^{n} u_{x x}+2 u^{n-1} u_{x}^{2}\right\} d x d s \\
\rightarrow \int_{0}^{t_{0}} \int_{\Omega} \chi_{\{u>0\}}\left\{-2 \kappa \psi_{x}(x) x^{\alpha-\kappa-1}+\psi_{x x}(x) x^{\alpha-\kappa}\right\}\left\{-u^{n} u_{x x}+2 u^{n-1} u_{x}^{2}\right\} d x d s .
\end{gathered}
$$

In combination with (4.27) and (4.28), in the limit $\eta \searrow 0$, this turns (4.26) into the identity

$$
\begin{aligned}
\int_{\Omega} \psi(x) x^{\beta-\kappa} & u\left(x, t_{0}\right) d x=\int_{\Omega} \psi(x) x^{\beta-\kappa} u_{0}(x) d x \\
& -\kappa(\kappa+1) \int_{0}^{t_{0}} \int_{\Omega} \chi_{\{u>0\}} \psi(x) x^{\alpha-\kappa-2} u^{n} u_{x x} d x d s \\
& +2 \kappa(\kappa+1) \int_{0}^{t_{0}} \int_{\Omega} \chi_{\{u>0\}} \psi(x) x^{\alpha-\kappa-2} u^{n-1} u_{x}^{2} d x d s \\
& +\int_{0}^{t_{0}} \int_{\Omega} \chi_{\{u>0\}}\left\{-2 \kappa \psi_{x}(x) x^{\alpha-\kappa-1}+\psi_{x x}(x) x^{\alpha-\kappa}\right\} \cdot\left\{-u^{n} u_{x x}+2 u^{n-1} u_{x}^{2} d x d s\right\} .
\end{aligned}
$$

Step 4. Let us reformulate (4.29) using generalized integration by parts.

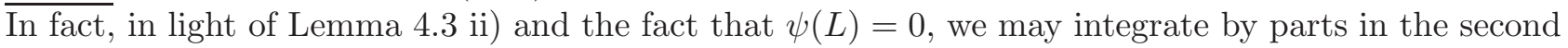
integral on the right-hand side of (4.29) so as to find that

$$
\begin{gathered}
-\kappa(\kappa+1) \int_{0}^{t_{0}} \int_{\Omega} \chi_{\{u>0\}} \psi(x) x^{\alpha-\kappa-2} u^{n} u_{x x} d x d s=n \kappa(\kappa+1) \int_{0}^{t_{0}} \int_{\Omega} \chi_{\{u>0\}} \psi(x) x^{\alpha-\kappa-2} u^{n-1} u_{x}^{2} d x d s \\
-\frac{\kappa(\kappa+1)(\alpha-\kappa-2)(\alpha-\kappa-3)}{n+1} \int_{0}^{t_{0}} \int_{\Omega} \psi(x) x^{\alpha-\kappa-4} u^{n+1} d x d s \\
+\kappa(\kappa+1) \int_{0}^{t_{0}} \int_{\Omega} \chi_{\{u>0\}} \psi_{x}(x) x^{\alpha-\kappa-2} u^{n} u_{x} d x d s \\
-\frac{\kappa(\kappa+1)(\alpha-\kappa-2)}{n+1} \int_{0}^{t_{0}} \int_{\Omega} \psi_{x}(x) x^{\alpha-\kappa-3} u^{n+1} d x d s .
\end{gathered}
$$

Consequently, (4.29) is equivalent to

$$
\begin{aligned}
\int_{\Omega} \psi(x) x^{\beta-\kappa} u(x, & \left.t_{0}\right) d x=\int_{\Omega} \psi(x) x^{\beta-\kappa} u_{0}(x) d x \\
+ & (n+2) \kappa(\kappa+1) \int_{0}^{t_{0}} \int_{\Omega} \chi_{\{u>0\}} \psi(x) x^{\alpha-\kappa-2} u^{n-1} u_{x}^{2} d x d s \\
& -\frac{\kappa(\kappa+1)(\alpha-\kappa-2)(\alpha-\kappa-3)}{n+1} \int_{0}^{t_{0}} \int_{\Omega} \psi(x) x^{\alpha-\kappa-4} u^{n+1} d x d s \\
& +\kappa(\kappa+1) \int_{0}^{t_{0}} \int_{\Omega} \chi_{\{u>0\}} \psi_{x}(x) x^{\alpha-\kappa-2} u^{n} u_{x} d x d s \\
& -\frac{\kappa(\kappa+1)(\alpha-\kappa-2)}{n+1} \int_{0}^{t_{0}} \int_{\Omega} \psi_{x}(x) x^{\alpha-\kappa-3} u^{n+1} d x d s \\
& +\int_{0}^{t_{0}} \int_{\Omega} \chi_{\{u>0\}}\left\{-2 \kappa \psi_{x}(x) x^{\alpha-\kappa-1}+\psi_{x x}(x) x^{\alpha-\kappa}\right\}\left\{-u^{n} u_{x x}+2 u^{n-1} u_{x}^{2}\right\} d x d s .
\end{aligned}
$$


Step 5. We finally derive the desired integral inequality (4.24).

For this purpose, we first note that the third term on the right-hand side of (4.30) can be related to the second one by means of (4.3), which is applicable again due to the fact that $\psi(L)=0$, and thus entails that

$$
\begin{gathered}
-\frac{\kappa(\kappa+1)(\alpha-\kappa-2)(\alpha-\kappa-3)}{n+1} \int_{0}^{t_{0}} \int_{\Omega} \psi(x) x^{\alpha-\kappa-4} u^{n+1} d x d s \\
\geq \quad-\frac{(n+1) \kappa(\kappa+1)(\alpha-\kappa-2)}{\alpha-\kappa-3} \int_{0}^{t_{0}} \int_{\Omega} \chi_{\{u>0\}} \psi(x) x^{\alpha-\kappa-2} u^{n-1} u_{x}^{2} d x d s \\
\quad+\frac{2 \kappa(\kappa+1)(\alpha-\kappa-2)}{n+1} \int_{0}^{t_{0}} \int_{\Omega} \psi_{x}(x) x^{\alpha-\kappa-3} u^{n+1} d x d s .
\end{gathered}
$$

Therefore, (4.30) yields

$$
\begin{aligned}
\int_{\Omega} \psi(x) x^{\beta-\kappa} & u\left(x, t_{0}\right) d x \geq \int_{\Omega} \psi(x) x^{\beta-\kappa} u_{0}(x) d x \\
& +\kappa(\kappa+1)\left\{n+2-\frac{(n+1)(\alpha-\kappa-2)}{\alpha-\kappa-3}\right\} \int_{0}^{t_{0}} \int_{\Omega} \chi_{\{u>0\}} \psi(x) x^{\alpha-\kappa-2} u^{n-1} u_{x}^{2} d x d s \\
& +\kappa(\kappa+1) \int_{0}^{t_{0}} \int_{\Omega} \chi_{\{u>0\}} \psi_{x}(x) x^{\alpha-\kappa-2} u^{n} u_{x} d x d s \\
& +\frac{\kappa(\kappa+1)(\alpha-\kappa-2)}{n+1} \int_{0}^{t_{0}} \int_{\Omega} \psi_{x}(x) x^{\alpha-\kappa-3} u^{n+1} d x d s \\
& +\int_{0}^{t_{0}} \int_{\Omega} \chi_{\{u>0\}}\left\{-2 \kappa \psi_{x}(x) x^{\alpha-\kappa-1}+\psi_{x x}(x) x^{\alpha-\kappa}\right\}\left\{-u^{n} u_{x x}+2 u^{n-1} u_{x}^{2}\right\} d x d s
\end{aligned}
$$

where thanks to (4.21) we know that

$$
c_{1}:=\kappa(\kappa+1)\left\{n+2-\frac{(n+1)(\alpha-\kappa-2)}{\alpha-\kappa-3}\right\}=\frac{\kappa(\kappa+1)(\alpha-\kappa-n-4)}{\alpha-\kappa-3}
$$

is positive. We can thus estimate the second and hence nonnegative summand on the right-hand side of (4.31) upon recalling that by construction, we have $0 \leq \psi \leq 1$ in $\Omega$ and $\psi \equiv 1$ in $\left(0, \frac{L}{4}\right)$, yielding

$$
\begin{aligned}
c_{1} \int_{0}^{t_{0}} \int_{\Omega} \chi_{\{u>0\}} \psi(x) x^{\alpha-\kappa-2} u^{n-1} u_{x}^{2} d x d s \geq & c_{1} \int_{0}^{t_{0}} \int_{\Omega} \chi_{\{u>0\}} x^{\alpha-\kappa-2} u^{n-1} u_{x}^{2} d x d s \\
& -c_{2} \int_{0}^{t_{0}} \int_{\frac{L}{4}}^{L} \chi_{\{u>0\}} u^{n-1} u_{x}^{2} d x d s
\end{aligned}
$$

for some $c_{2}>0$ which, as well as $c_{3}, c_{4}, \ldots$ below, neither depends on $t_{0}$ nor on $u$.

Next, since $\psi_{x} \equiv \psi_{x x} \equiv 0$ in $\left(0, \frac{L}{4}\right)$, we can find $c_{3}>0$ such that

$$
\left|\kappa(\kappa+1) \int_{0}^{t_{0}} \int_{\Omega} \chi_{\{u>0\}} \psi_{x}(x) x^{\alpha-\kappa-2} u^{n} u_{x} d x d s\right| \leq c_{3} \int_{0}^{t_{0}} \int_{\frac{L}{4}}^{L} \chi_{\{u>0\}} u^{n}\left|u_{x}\right| d x d s
$$

and

$$
\left|\frac{\kappa(\kappa+1)(\alpha-\kappa-2)}{n+1} \int_{0}^{t_{0}} \int_{\Omega} \psi_{x}(x) x^{\alpha-\kappa-3} u^{n+1} d x d s\right| \leq c_{3} \int_{0}^{t_{0}} \int_{\frac{L}{4}}^{L} u^{n+1} d x d s
$$


as well as

$$
\begin{gathered}
\left|\int_{0}^{t_{0}} \int_{\Omega} \chi_{\{u>0\}}\left\{-2 \kappa \psi_{x}(x) x^{\alpha-\kappa-1}+\psi_{x x}(x) x^{\alpha-\kappa}\right\}\left\{-u^{n} u_{x x}+2 u^{n-1} u_{x}^{2}\right\} d x d s\right| \\
\leq c_{3} \int_{0}^{t_{0}} \int_{\frac{L}{4}}^{L} \chi_{\{u>0\}} u^{n}\left|u_{x x}\right| d x d s+c_{3} \int_{0}^{t_{0}} \int_{\frac{L}{4}}^{L} \chi_{\{u>0\}} u^{n-1} u_{x}^{2} d x d s .
\end{gathered}
$$

Since by the Cauchy-Schwarz inequality,

$$
\int_{0}^{t_{0}} \int_{\frac{L}{4}}^{L} \chi_{\{u>0\}} u^{n}\left|u_{x}\right| d x d s \leq \frac{1}{2} \int_{0}^{t_{0}} \int_{\frac{L}{4}}^{L} \chi_{\{u>0\}} u^{n-1} u_{x}^{2} d x d s+\frac{1}{2} \int_{0}^{t_{0}} \int_{\frac{L}{4}}^{L} u^{n+1} d x d s,
$$

and since $\psi \equiv 1$ in $\left(0, \frac{L}{4}\right)$ and $0 \leq \psi \leq 1$ imply that

$$
\int_{\Omega} \psi(x) x^{\beta-\kappa} u_{0}(x) d x \geq \int_{0}^{\frac{L}{4}} x^{\beta-\kappa} u_{0}(x) d x
$$

and

$$
\int_{\Omega} \psi(x) x^{\beta-\kappa} u\left(x, t_{0}\right) d x \leq y\left(t_{0}\right)
$$

with $y$ as defined in (4.23), (4.31) therefore shows that

$$
\begin{aligned}
y\left(t_{0}\right) \geq & \int_{0}^{\frac{L}{4}} x^{\beta-\kappa} u_{0}(x) d x+c_{1} \int_{0}^{t_{0}} \int_{\Omega} \chi_{\{u>0\}} x^{\alpha-\kappa-2} u^{n-1} u_{x}^{2} d x d s \\
& -c_{3} \int_{0}^{t_{0}} \int_{\frac{L}{4}}^{L} \chi_{\{u>0\}} u^{n}\left|u_{x x}\right| d x d s-\left(c_{2}+\frac{3}{2} c_{3}\right) \int_{0}^{t_{0}} \int_{\frac{L}{4}}^{L} \chi_{\{u>0\}} u^{n-1} u_{x}^{2} d x d s \\
& -\frac{3}{2} c_{3} \int_{0}^{t_{0}} \int_{\frac{L}{4}}^{L} u^{n+1} d x d s .
\end{aligned}
$$

Now thanks to (4.21), the interpolation inequality privided by Lemma 2.2 becomes applicable such that with some $c_{4}>0$, we have

$$
\int_{\Omega} \chi_{\{u>0\}} x^{\alpha-\kappa-2} u^{n-1} u_{x}^{2} d x \geq\left\{c_{4} \int_{\Omega} x^{\beta-\kappa} u(x, t) d x-\int_{\Omega} x^{\beta} u(x, t) d x\right\}_{+}^{n+1},
$$

which in view of (3.4) means that there exists $c_{5}>0$ and $c_{6}>0$ such that the second integral on the right-hand side of (4.36) can be estimated as

$$
c_{1} \int_{0}^{t_{0}} \int_{\Omega} \chi_{\{u>0\}} x^{\alpha-\kappa-2} u^{n-1} u_{x}^{2} d x d s \geq c_{5} \int_{0}^{t_{0}}\left(y(s)-c_{6} B\right)_{+}^{n+1} d s .
$$

Now, the nonpositive terms on the right-hand side of (4.36) can be controlled by using the entropy inequality (3.6) through its consequences stated in Lemma 4.1. To prepare this, we first invoke the Cauchy-Schwarz inequality to estimate

$$
\int_{0}^{t_{0}} \int_{\frac{L}{4}}^{L} \chi_{\{u>0\}} u^{n}\left|u_{x x}\right| d x d s \leq\left(\int_{0}^{t_{0}} \int_{\frac{L}{4}}^{L} \chi_{\{u>0\}} u^{n-2} u_{x x}^{2} d x d s\right)^{\frac{1}{2}}\left(\int_{0}^{t_{0}} \int_{\frac{L}{4}}^{L} u^{n+2} d x d s\right)^{\frac{1}{2}},
$$


and then apply Lemma 2.4 to $\Omega_{0}=\left(\frac{L}{4}, L\right)$ and $p=n+2$ to find $c_{7}>0$ satisfying

$$
\begin{aligned}
c_{3} \int_{0}^{t_{0}} \int_{\frac{L}{4}}^{L} \chi_{\{u>0\}} u^{n}\left|u_{x x}\right| d x d s \\
\leq c_{7}\left(\int_{0}^{t_{0}} \int_{\frac{L}{4}}^{L} \chi_{\{u>0\}} u^{n-2} u_{x x}^{2} d x d s\right)^{\frac{1}{2}} \\
\times \\
\times\left\{\int_{0}^{t_{0}}\left(\int_{\frac{L}{4}}^{L} u d x\right)^{\frac{2(2 n+3)}{n+3}}\left(\int_{\frac{L}{4}}^{L} \chi_{\{u>0\}} u^{n-4} u_{x}^{4} d x\right)^{\frac{n+1}{n+3}} d s+\int_{0}^{t_{0}}\left(\int_{\frac{L}{4}}^{L} u d x\right)^{n+2} d s\right\}^{\frac{1}{2}} .
\end{aligned}
$$

Here, since $\frac{L}{4}$ is positive, again using (3.4), we see that

$$
\int_{\frac{L}{4}}^{L} u d x \leq c_{8} \int_{\frac{L}{4}}^{L} x^{\beta} u d x \leq c_{8} \int_{\Omega} x^{\beta} u d x=c_{8} \int_{\Omega} x^{\beta} u_{0} d x \leq c_{8} B
$$

with $c_{8}:=\left(\frac{L}{4}\right)^{-\beta}$, and similarly, we find that

$$
\int_{\frac{L}{4}}^{L} \chi_{\{u>0\}} u^{n-4} u_{x}^{4} d x \leq c_{9} \int_{\Omega} \chi_{\{u>0\}} x^{\alpha} u^{n-4} u_{x}^{4} d x
$$

and

$$
\int_{\frac{L}{4}}^{L} \chi_{\{u>0\}} u^{n-2} u_{x x}^{2} d x \leq c_{9} \int_{\Omega} \chi_{\{u>0\}} x^{\alpha} u^{n-2} u_{x x}^{2} d x
$$

hold with $c_{9}:=\left(\frac{L}{4}\right)^{-\alpha}$. Accordingly, (4.38) implies that for some $c_{10}(B)>0$, we have

$$
\begin{aligned}
c_{3} \int_{0}^{t_{0}} \int_{\frac{L}{4}}^{L} \chi_{\{u>0\}} u^{n}\left|u_{x x}\right| d x d s \leq & c_{10}(B)\left(\int_{0}^{t_{0}} \int_{\Omega} \chi_{\{u>0\}} x^{\alpha} u^{n-2} u_{x x}^{2} d x d s\right)^{\frac{1}{2}} \times \\
& \times\left\{\int_{0}^{t_{0}}\left(\int_{\Omega} \chi_{\{u>0\}} x^{\alpha} u^{n-4} u_{x}^{4} d x\right)^{\frac{n+1}{n+3}} d s+t_{0}\right\}^{\frac{1}{2}} .
\end{aligned}
$$

By Lemma 4.1, we can find $c_{11}(B, D)>0$ such that

$$
\int_{0}^{t_{0}} \int_{\Omega} \chi_{\{u>0\}} x^{\alpha} u^{n-2} u_{x x}^{2} d x d s+\int_{0}^{t_{0}} \int_{\Omega} \chi_{\{u>0\}} x^{\alpha} u^{n-4} u_{x}^{4} d x d s \leq c_{11}(B, D) .
$$

Therefore, since by the Hölder inequality we know that

$$
\int_{0}^{t_{0}}\left(\int_{\Omega} \chi_{\{u>0\}} x^{\alpha} u^{n-4} u_{x}^{4} d x\right)^{\frac{n+1}{n+3}} d s \leq\left(\int_{0}^{t_{0}} \int_{\Omega} \chi_{\{u>0\}} x^{\alpha} u^{n-4} u_{x}^{4} d x d s\right)^{\frac{n+1}{n+3}} t_{0}^{\frac{2}{n+3}},
$$

from (4.39) and Young's inequality, we obtain

$$
\begin{aligned}
c_{3} \int_{0}^{t_{0}} \int_{\frac{L}{4}}^{L} \chi_{\{u>0\}} u^{n}\left|u_{x x}\right| d x d s & \leq c_{10}(B) \\
& \times\left\{\left(\int_{0}^{t_{0}} \int_{\Omega} \chi_{\{u>0\}} x^{\alpha} u^{n-2} u_{x x}^{2} d x d s\right)^{\frac{1}{2}} \times\right. \\
& \left.\left.\leq \int_{\Omega}^{t_{0}} \chi_{\{u>0\}} x^{\alpha} u^{n-4} u_{x}^{4} d x d s\right)^{\frac{n+1}{n+3}} t_{0}^{\frac{2}{n+3}}+t_{0}\right\}^{\frac{1}{2}} \\
& c_{12}(B, D)(1+T)
\end{aligned}
$$


for some $c_{12}(B, D)>0$, because $t_{0} \leq T$.

Similarly, as for the second to last term in (4.36), we first find that

$$
\int_{0}^{t_{0}} \int_{\frac{L}{4}}^{L} \chi_{\{u>0\}} u^{n-1} u_{x}^{2} d x d s \leq\left(\int_{0}^{t_{0}} \int_{\frac{L}{4}}^{L} \chi_{\{u>0\}} u^{n-4} u_{x}^{4} d x d s\right)^{\frac{1}{2}}\left(\int_{0}^{t_{0}} \int_{\frac{L}{4}}^{L} u^{n+2} d x d s\right)^{\frac{1}{2}},
$$

and then again apply Lemma 2.4 and (4.40) to estimate

$$
\begin{aligned}
\left(c_{2}+\frac{3}{2} c_{3}\right) \int_{0}^{t_{0}} \int_{\frac{L}{4}}^{L} \chi_{\{u>0\}} u^{n-1} u_{x}^{2} d x d s \leq & c_{13}(B)\left(\int_{0}^{t_{0}} \int_{\Omega} \chi_{\{u>0\}} x^{\alpha} u^{n-4} u_{x}^{4} d x d s\right)^{\frac{1}{2}} \times \\
& \times\left\{\int_{0}^{t_{0}}\left(\int_{\Omega} \chi_{\{u>0\}} x^{\alpha} u^{n-4} u_{x}^{4} d x\right)^{\frac{n+1}{n+3}} d s t_{0}^{\frac{2}{n+3}}+t_{0}\right\}^{\frac{1}{2}} \\
\leq & c_{14}(B, D) \cdot(1+T)
\end{aligned}
$$

for suitable $c_{13}(B)>0$ and $c_{14}(B, D)>0$.

Finally, in much the same manner, the last term in (4.36) can be controlled. Indeed, again on the basis of Lemma 2.4, this time applied to $p=n+1$, we can use (4.40) to find positive constants $c_{15}(B)$ and $c_{16}(B, D)$ such that

$$
\begin{aligned}
\frac{3}{2} c_{3} \int_{0}^{t_{0}} \int_{\frac{L}{4}}^{L} u^{n+1} d x d s & \leq c_{15}(B)\left\{\int_{0}^{t_{0}}\left(\int_{\frac{L}{4}}^{L} \chi_{\{u>0\}} u^{n-4} u_{x}^{4} d x\right)^{\frac{n}{n+3}} d s+t_{0}\right\} \\
& \leq c_{15}(B)\left\{\left(\int_{0}^{t_{0}} \int_{\Omega} \chi_{\{u>0\}} x^{\alpha} u^{n-4} u_{x}^{4} d x\right)^{\frac{n}{n+3}} d s t_{0}^{\frac{3}{n+3}}+t_{0}\right\} \\
& \leq c_{16}(B, D)(1+T) .
\end{aligned}
$$

Combining (4.36) and (4.37) with (4.41)-(4.43), we therefore arrive at (4.24).

\subsection{Blow-up. Proof of Theorem 1.2 and Proposition 1.3}

The above inequality (4.24) can now be turned into a sufficient condition for blow-up by means of the following variant of Gronwall's lemma.

Lemma 4.5 (Nonlinear Gronwall lemma) Let $a>0, b>0, d>0$, and $m>1$ be such that

$$
a>2 d,
$$

and suppose that for some $T>0, y \in C^{0}([0, T])$ is nonnegative and satisfies

$$
y(t) \geq a+b \int_{0}^{t}(y(s)-d)_{+}^{m} d s \quad \text { for all } t \in[0, T] .
$$

Then

$$
T<\frac{2^{m}}{(m-1) b a^{m-1}}
$$


Proof. For $\delta \in(0, a-2 d)$, let $z_{\delta}$ be the solution of the initial-value problem

$$
\left\{\begin{array}{l}
z_{\delta}^{\prime}(t)=2^{-m} b \cdot z_{\delta}^{m}(t), \quad t \in\left(0, T_{\delta}\right), \\
z_{\delta}(0)=a-\delta
\end{array}\right.
$$

defined up to its maximal existence time $T_{\delta}>0$; that is, we let

$$
z_{\delta}(t):=\left\{(a-\delta)^{1-m}-\frac{(m-1) b}{2^{m}} \cdot t\right\}^{-\frac{1}{m-1}}, \quad t \in\left[0, T_{\delta}\right)
$$

with

$$
T_{\delta}:=\frac{2^{m}}{(m-1) b(a-\delta)^{m-1}} .
$$

Then $z_{\delta}^{\prime} \geq 0$ and thus $z_{\delta} \geq a-\delta \geq 2 d$ on $\left(0, T_{\delta}\right)$, so that

$$
z_{\delta}^{\prime}=b\left(z_{\delta}-\frac{z_{\delta}}{2}\right)^{m} \leq b\left(z_{\delta}-d\right)^{m}=b\left(z_{\delta}-d\right)_{+}^{m} \quad \text { for all } t \in\left(0, T_{\delta}\right)
$$

and hence

$$
z_{\delta}(t) \leq a-\delta+b \int_{0}^{t}\left(z_{\delta}(s)-d\right)_{+}^{m} d s \quad \text { for all } t \in\left[0, T_{\delta}\right) .
$$

Now, by (4.44) and the continuity of $y$ and $z_{\delta}$, the number

$$
t_{0}:=\sup \left\{t \in\left(0, T_{\delta}\right) \mid y>z_{\delta} \text { in }[0, t]\right\}
$$

is well-defined. However, if $t_{0}$ was smaller than $T_{\delta}$ then $y\left(t_{0}\right)=z_{\delta}\left(t_{0}\right)$, and therefore (4.45) and (4.50) would yield

$$
\begin{aligned}
z_{\delta}\left(t_{0}\right) & =y\left(t_{0}\right) \geq a+b \int_{0}^{t_{0}}(y(s)-d)_{+}^{m} d s \geq a+b \int_{0}^{t_{0}}\left(z_{\delta}(s)-d\right)_{+}^{m} d s \\
& >a-\delta+b \int_{0}^{t_{0}}\left(z_{\delta}(s)-d\right)_{+}^{m} d s \geq z_{\delta}\left(t_{0}\right),
\end{aligned}
$$

which is absurd. We thus have $t_{0}=t_{\delta}$ and hence $y>z_{\delta}$ on $\left[0, T_{\delta}\right)$ for all $\delta \in(0, a-2 d)$. In view of (4.48) and (4.49), in the limit $\delta \searrow 0$, this implies (4.46).

We can now pass to the proof of our main result of blow-up in (1.1).

Proof of Theorem 1.2. Given $B>0$ and $D>0$, we let $C_{1}(B . D), C_{2}$, and $C_{3}$ denote the positive constants provided by Lemma 4.4. For fixed $T>0$, we then choose a large number $M>0$ fulfilling

$$
M \geq 2\left(\frac{L}{4}\right)^{-\kappa} B
$$

and

$$
M \geq 4 C_{1}(B, D)(1+T)
$$

as well as

$$
M>8 C_{3} B
$$


and

$$
M \geq 4\left(\frac{2^{n+1}}{n C_{2} T}\right)^{\frac{1}{n}}
$$

Now, assuming that $u$ were any entropy solution of (1.1) in $\Omega \times(0, T)$ with some nonnegative $u_{0} \in$ $C^{0}(\bar{\Omega})$ fulfilling

$$
\int_{\Omega} x^{\beta} u_{0}(x) d x \leq B
$$

and

$$
\int_{\Omega} x^{\beta} \ln _{+} \frac{1}{u_{0}(x)} d x \leq D
$$

as well as

$$
\int_{\Omega} x^{\beta-\kappa} u_{0}(x) d x \geq M
$$

we would obtain from Lemma 4.4 that $y(t):=\int_{\Omega} x^{\beta-\kappa} u(x, t) d x, t \in[0, T)$, satisfies

$$
y(t) \geq \int_{0}^{\frac{L}{4}} x^{\beta-\kappa} u_{0}(x) d x-C_{1}(B, D)(1+T)+C_{2} \int_{0}^{t}\left(y(s)-C_{3} B\right)_{+}^{n+1} d s \quad \text { for all } t \in(0, T) .
$$

Here, we first use (4.57), (4.55), and (4.51) to estimate

$$
\begin{aligned}
\int_{0}^{\frac{L}{4}} x^{\beta-\kappa} u_{0}(x) d x & =\int_{\Omega} x^{\beta-\kappa} u_{0}(x) d x-\int_{\frac{L}{4}}^{L} x^{\beta-\kappa} u_{0}(x) d x \\
& \geq M-\left(\frac{L}{4}\right)^{-\kappa} \int_{\frac{L}{4}}^{L} x^{\beta} u_{0}(x) d x \geq M-\left(\frac{L}{4}\right)^{-\kappa} B \geq \frac{M}{2},
\end{aligned}
$$

and then invoke (4.52) to see that

$$
-C_{1}(B, D)(1+T) \geq-\frac{M}{4}
$$

Thereupon, (4.58) implies that

$$
y(t) \geq \frac{M}{4}+C_{2} \int_{0}^{t}\left(y(s)-C_{3} B\right)_{+}^{n+1} d s \quad \text { for all } t \in(0, T),
$$

so that, since $\frac{M}{4}>2 C_{3} B$ by (4.53), Lemma 4.5 becomes applicable so as to show that necessarily

$$
T<\frac{2^{n+1}}{n C_{2} \cdot\left(\frac{M}{4}\right)^{n}}
$$

In view of (4.54), however, we have

$$
\frac{2^{n+1}}{n C_{2} \cdot\left(\frac{M}{4}\right)^{n}} \leq \frac{2^{n+1}}{n C_{2} \cdot \frac{2^{n+1}}{n C_{2} T}}=T
$$

which proves that in fact such a solution cannot exist. 
Now since from (1.4), we already know that the maximally extended entropy solution constructed in Theorem 1.1 can cease to exist in finite time only when (1.8) holds, the proof is complete.

Proof of Proposition 1.3. Since $\kappa>0$ and $\kappa<\beta+1$, given $p<\frac{1}{\beta+1-\kappa}$, we can pick a positive number $\theta$ fulfilling $\beta+1-\kappa<\theta<\beta+1$ as well as $p \theta<1$. We then choose a nonnegative nontrivial $\varphi \in C_{0}^{\infty}\left((0, \infty)\right.$ such that $\operatorname{supp} \varphi \subset \Omega$, and for any $u_{0}$ with the indicated properties, we let

$$
u_{0 k}(x):=u_{0}(x)+k^{\theta} \varphi(k x)
$$

for $x \in \Omega$ and $k \in \mathbb{N}$. Then clearly, $u_{0 k}-u_{0}$ belongs to $C_{0}^{\infty}(\Omega)$ for all $k \in \mathbb{N}$, and by direct computation we see that

$$
\begin{aligned}
\int_{\Omega} x^{\beta} u_{0 k}(x) d x & =\int_{\Omega} x^{\beta} u_{0}(x) d x+k^{\theta} \int_{\Omega} x^{\beta} \varphi(k x) d x \\
& =\int_{\Omega} x^{\beta} u_{0}(x) d x+k^{\theta-\beta-1} \int_{0}^{k L} y^{\beta} \varphi(y) d y \\
& \rightarrow \int_{\Omega} x^{\beta} u_{0}(x) d x \quad \text { as } k \rightarrow \infty,
\end{aligned}
$$

because $\theta<\beta+1$. Similarly, using $\theta>\beta+1-\kappa$, we obtain

$$
\begin{aligned}
\int_{\Omega} x^{\beta-\kappa} u_{0 k}(x) d x & =\int_{\Omega} x^{\beta-\kappa} u_{0}(x) d x+k^{\theta} \int_{\Omega} x^{\beta-\kappa} \varphi(k x) d x \\
& =\int_{\Omega} x^{\beta-\kappa} u_{0}(x) d x+k^{\theta-\beta+\kappa-1} \int_{0}^{k L} y^{\beta-\kappa} \varphi(y) d y \\
& \rightarrow \infty \quad \text { as } k \rightarrow \infty,
\end{aligned}
$$

whereas the inequality $p \theta<1$ asserts that

$$
\left\|u_{0 k}-u_{0}\right\|_{L^{p}(\Omega)}^{p}=k^{p \theta} \int_{\Omega} \varphi^{p}(k x) d x=k^{p \theta-1} \int_{0}^{k L} \varphi^{p}(y) d y \rightarrow 0 \quad \text { as } k \rightarrow \infty .
$$

Finally, since clearly $u_{0 k} \rightarrow u_{0}$ in the pointwise sense in $\Omega$, and since $x^{\beta} \ln _{+} \frac{1}{u_{0 k}} \leq x^{\beta} \ln _{+} \frac{1}{u_{0}}$ in $\Omega$ by the nonnegativity of $\varphi$ and our assumption that $x^{\beta} \ln _{+} \frac{1}{u_{0}}$ is integrable, the dominated convergence theorem shows that also (1.11) holds.

\section{A Appendix: Regularization of the initial data}

Let us finally make sure that it is in fact possible to approximate initial data by smooth functions in the sense referred to in the proof of Theorem 1.2, Indeed, the following elementary construction shows that the requirements (3.28)-(3.31) can be fulfilled simultaneously.

Lemma A.1 (Regularization of initial data) Let $\beta>-1, \gamma<1$, and $u \in W_{\gamma}^{1,2}(\Omega)$ be nonnegative such that

$$
\int_{\Omega} x^{\beta} \ln u(x) d x>-\infty
$$


Then, given any $\left(\varepsilon_{j}\right)_{j \in \mathbb{N}} \subset(0, \infty)$ such that $\varepsilon_{j} \searrow 0$ as $j \rightarrow \infty$, one can find $\left(u_{j}\right)_{j \in \mathbb{N}} \subset C^{\infty}(\bar{\Omega})$ such that

$$
u_{j}>0 \quad \text { in } \bar{\Omega} \quad \text { and } \quad u_{j x} \in C_{0}^{\infty}(\Omega) \quad \text { for all } j \in \mathbb{N}
$$

and

$$
u_{j} \rightarrow u \quad \text { in } C^{0}(\bar{\Omega})
$$

as well as

$$
\int_{\Omega}\left(x+\varepsilon_{j}\right)^{\gamma} u_{j x}^{2}(x) d x \rightarrow \int_{\Omega} x^{\gamma} u_{x}^{2}(x) d x
$$

and

$$
\int_{\Omega}\left(x+\varepsilon_{j}\right)^{\beta} \ln u_{j}(x) d x \rightarrow \int_{\Omega} x^{\beta} \ln u(x) d x
$$

as $j \rightarrow \infty$.

Proof. $\quad$ Since $\gamma<1$ and hence $W_{\gamma}^{1,2}(\Omega) \hookrightarrow C^{0}(\bar{\Omega})$, we know that $M:=\|u\|_{L^{\infty}(\Omega)}+1$ is finite. Given $\left(\varepsilon_{j}\right)_{j \in \mathbb{N}} \subset(0, \infty)$ with $\varepsilon_{j} \searrow 0$ as $j \rightarrow \infty$, we let

$$
\eta_{j}:=\left(\int_{\Omega}\left|\left(x+\varepsilon_{j}\right)^{-\frac{\gamma}{2}}-x^{-\frac{\gamma}{2}}\right|^{2}\right)^{\frac{1}{2}} \cdot\left(\int_{\Omega} x^{\gamma} u_{x}^{2}\right)^{\frac{1}{2}}, \quad j \in \mathbb{N},
$$

so that again, since $\gamma<1$, the Beppo-Levi theorem asserts that $\eta_{j} \searrow 0$ as $j \rightarrow \infty$. Accordingly, the numbers

$$
\delta_{j}:= \begin{cases}4 \eta_{j} & \text { if } \beta \leq 0, \\ \max \left\{4 \eta_{j}, 2 M e^{-\varepsilon_{j}^{-\beta}}\right\} & \text { if } \beta>0,\end{cases}
$$

also satisfy $\delta_{j} \searrow 0$ as $j \rightarrow \infty$.

Once more using that $x^{\frac{\gamma}{2}} u_{x}(x)$ belongs to $L^{2}(\Omega)$, for each $j \in \mathbb{N}$, by density, we can find $v_{j} \in C_{0}^{\infty}(\Omega)$ such that

$$
\int_{\Omega}\left|v_{j}(x)-x^{\frac{\gamma}{2}} u_{x}(x)\right|^{2}<\left(\frac{\delta_{j}}{4 c_{1}}\right)^{2}
$$

where

$$
c_{1}:=\sup _{j \in \mathbb{N}}\left(\int_{\Omega}\left(x+\varepsilon_{j}\right)^{-\gamma} d x\right)^{\frac{1}{2}} .
$$

We now define

$$
u_{j}(x):=u(0)+\delta_{j}+\int_{0}^{x}\left(y+\varepsilon_{j}\right)^{-\frac{\gamma}{2}} v_{j}(y) d y \quad \text { for } x \in \bar{\Omega} \text { and } j \in \mathbb{N} .
$$

Then clearly $u_{j} \in C^{\infty}(\bar{\Omega})$ with $u_{j x} \in C_{0}^{\infty}(\Omega)$, and using the Cauchy-Schwarz inequality, (1.66), and (1.64), we can estimate

$$
\begin{aligned}
\left|\int_{0}^{x} u_{j x}(y) d y-\int_{0}^{x} u_{x}(y) d y\right|= & \mid \int_{0}^{x}\left(y+\varepsilon_{j}\right)^{-\frac{\gamma}{2}}\left\{v_{j}(y)-y^{\frac{\gamma}{2}} u_{x}(y)\right\} d y \\
& +\int_{0}^{x}\left\{\left(y+\varepsilon_{j}\right)^{-\frac{\gamma}{2}}-y^{-\frac{\gamma}{2}}\right\} \cdot y^{\frac{\gamma}{2}} u_{x}(y) d y \mid
\end{aligned}
$$




$$
\begin{aligned}
\leq & \left(\int_{\Omega}(y+\varepsilon)^{-\gamma} d y\right)^{\frac{1}{2}} \cdot\left(\int_{\Omega}\left|v_{j}(y)-y^{\frac{\gamma}{2}} u_{x}(y)\right|^{2} d y\right)^{\frac{1}{2}} \\
& +\left(\int_{\Omega}\left|\left(y+\varepsilon_{j}\right)^{-\frac{\gamma}{2}}-y^{-\frac{\gamma}{2}}\right|^{2} d y\right)^{\frac{1}{2}}\left(\int_{\Omega} y^{\gamma} u_{x}^{2}(y) d y\right)^{\frac{1}{2}} \\
\leq & c_{1} \frac{\delta_{j}}{4 c_{1}}+\eta_{j} \\
\leq & \frac{\delta_{j}}{2} \quad \text { for all } x \in \Omega \text { and } j \in \mathbb{N},
\end{aligned}
$$

because $\eta_{j} \leq \frac{\delta_{j}}{4}$ by (1.65). In particular, this implies that

$$
u_{j}(x)-u(x)=\delta_{j}+\int_{0}^{x} u_{j x}(y) d y-\int_{0}^{x} u_{x}(y) d y \geq \frac{\delta_{j}}{2} \quad \text { for all } x \in \Omega \text { and } j \in \mathbb{N},
$$

and similarly,

$$
u_{j}(x)-u(x) \leq \frac{3 \delta_{j}}{2} \quad \text { for all } x \in \Omega \text { and } j \in \mathbb{N},
$$

which, since $\delta_{j} \searrow 0$ as $j \rightarrow \infty$, proves (1.61). Moreover, it is clear from (1.67) and (1.66) that

$$
\int_{\Omega}\left(x+\varepsilon_{j}\right)^{\gamma} u_{j x}^{2}(x) d x=\int_{\Omega} v_{j}^{2}(x) d x \rightarrow \int_{\Omega} x^{\gamma} u_{x}^{2}(x) d x \quad \text { as } j \rightarrow \infty,
$$

whence it remains to show (1.63). For this purpose, thanks to (1.61), we may pick $j_{0} \in \mathbb{N}$ such that $u_{j} \leq M$ in $\Omega$ for all $j \geq j_{0}$, which guarantees that $\ln \frac{M}{u_{j}} \geq 0$ in $\Omega$ for all such $j$.

Then in the case $\beta \leq 0$, we use the fact that (1.68) entails that $u_{j} \geq u$ in estimating

$$
\left(x+\varepsilon_{j}\right)^{\beta} \ln \frac{M}{u_{j}(x)} \leq x^{\beta} \ln \frac{M}{u(x)} \quad \text { for all } x \in \Omega \text { and } j \geq j_{0},
$$

so that by (1.59) and the dominated convergence theorem, we infer that

$$
\int_{\Omega}\left(x+\varepsilon_{j}\right)^{\beta} \ln \frac{M}{u_{j}(x)} d x \rightarrow \int_{\Omega} x^{\beta} \ln \frac{M}{u(x)} d x \quad \text { as } j \rightarrow \infty,
$$

which clearly implies (1.63) in this case.

When $\beta>0$, we first use the pointwise estimate $\left(x+\varepsilon_{j}\right)^{\beta} \leq 2^{\beta}\left(x^{\beta}+\varepsilon_{j}^{\beta}\right)$ for $x \in \Omega$ and $j \in \mathbb{N}$ and then observe that (1.68) entails that $u_{j} \geq \frac{\delta_{j}}{2}$ in $\Omega$ for all $j \in \mathbb{N}$ to see that

$$
\begin{aligned}
\left(x+\varepsilon_{j}\right)^{\beta} \ln \frac{M}{u_{j}(x)} & \leq 2^{\beta} x^{\beta} \ln \frac{M}{u(x)}+2^{\beta} \varepsilon_{j}^{\beta} \ln \frac{2 M}{\delta_{j}} \\
& \leq 2^{\beta} x^{\beta} \ln \frac{M}{u(x)}+2^{\beta} \quad \text { for all } x \in \Omega \text { and } j \geq j_{0}
\end{aligned}
$$

according to our choice (1.65) of $\delta_{j}$. Again by (1.59) and dominated convergence, this yields (1.69) and hence (1.63) also in this case.

Acknowledgement. The first author acknowledges partial support from the Austrian Science Fund (FWF), grants P20214, P22108, I395, and W1245. 


\section{References}

[1] A. Bertozzi and M. Pugh. Finite-time blow-up of solutions of some long-wave unstable thin film equations. Indiana Univ. Math. J. 49 (2000), 1323-1366.

[2] J. A. Carrillo, M. Di Francesco, G. Toscani. Condensation phenomena in nonlinear drift equations. Preprint, 2013. arXiv:1307.2275.

[3] J. A. Carrillo, A. Jüngel, P. Markowich, G. Toscani, and A. Unterreiter. Entropy dissipation methods for degenerate parabolic problems and generalized Sobolev inequalities. Monatsh. Math. 133 (2001), 1-82.

[4] M. Chaves, V.A. Galaktionov. Regional blow-up for a higher-order semilinear parabolic equation. Eur. J. Appl. Math. 12 (2001), 601-623.

[5] M. Escobedo, M. Herrero, and J. Velázquez. A nonlinear Fokker-Planck equation modelling the approach to thermal equilibrium in a homogeneous plasma. Trans. Amer. Math. Soc. 350 (1998), 3837-3901.

[6] M. Escobedo and J. Velázquez. Finite time blow-up for the bosonic Nordheim equation. Preprint, 2012. arXiv:1206.5410.

[7] M. Escobedo and J. Velázquez. On the blow up of supercritical solution of the Nordheim equation for bosons. Preprint, 2012. 1210.1664.

[8] V.A. Galaktionov, E. Mitidieri, and S.I. Pohozaev. On global solutions and blow-up for KuramotoSivashinsky-type models, and well-posed Burnett equations Nonlinear Analysis 70 (2009), 29302952.

[9] R. Glassey. On the blowing up of solutions to the Cauchy problem for nonlinear Schrödinger equations. J. Math. Phys. 18 (1977), 1794-1797.

[10] K. Ishige. Blow-up time and blow-up set of the solutions for semilinear heat equations with large diffusion. Adv. Diff. Eqs. 7 (2002), 1003-1024.

[11] C. Josserand, Y. Pomeau, and S. Rica. Self-similar singularities in the kinetics of condensation. J. Low Temp. Phys. 145 (2006), 231-265.

[12] A. Jüngel and R. Weishäupl. Blow-up in two-component nonlinear Schrödinger systems with an external driven field. Math. Models Meth. Appl. Sci. 23 (2013), 1699-1727.

[13] A. Jüngel and M. Winkler. A degenerate fourth-order parabolic equation modeling Bose-Einstein condensation. Part I: Local existence of solutions. Preprint, 2013.

[14] G. Kaniadakis and P. Quarati. Kinetic equation for classical particles obeying an exclusion principle. Phys. Rev. E 48 (1993), 4263-4270.

[15] S. Kaplan. On the growth of solutions of quasilinear parabolic equations. Comm. Pure Appl. Math. 16 (1963), 305330. 
[16] A. Kompaneets. The establishment of thermal equilibrium between quanta and electrons. Soviet Physics JETP 4 (1957), 730-737.

[17] H.A. Levine. The role of critical exponents in blowup theorems. SIAM Rev. 32 (1990), 262288.

[18] P. Quitnner and P. Souplet. Superlinear parabolic problems. Blow-up, global existence and steady states. Birkhäuser Advanced Texts, Basel, 2007.

[19] G. Toscani. Finite time blow up in Kaniadakis-Quarati model of Bose-Einstein particles. Commun. Part. Diff. Eqs. 37 (2012), 77-87.

[20] M. Winkler. Finite-time blow-up in the higher-dimensional parabolic-parabolic Keller-Segel system. J. Math. Pures Appl. 100 (2013), 748-767. arXiv:1112.4156v1 